\title{
Probing the structure of protoplanetary disks: a comparative study of DM Tau, LkCa 15, and MWC 480
}

\author{
V. Piétu ${ }^{1,2}$, A. Dutrey ${ }^{1}$, and S. Guilloteau ${ }^{1}$ \\ 1 Université Bordeaux 1, CNRS, OASU, UMR 5804, BP 89, 2 rue de l'Observatoire, 33270 Floirac, France \\ e-mail: pietu@iram.fr \\ 2 Institut de Radio-Astronomie Millimétrique, 300 rue de la Piscine, Domaine Universitaire, 38406 Saint Martin d'Hères, France
}

Received 10 October 2006 / Accepted 8 January 2007

\section{ABSTRACT}

\begin{abstract}
Context. The physical structure of proto-planetary disks is not yet well constrained by current observations. Millimeter interferometry is an essential tool to investigate young disks.

Aims. We study the vertical and radial temperature distribution in a few well-known disks from an observational perspective. The surface density distribution of $\mathrm{CO}$ and $\mathrm{HCO}^{+}$and the scale-height are also investigated.

Methods. We report CO observations at sub-arcsecond resolution with the IRAM array of the disks surrounding MWC 480, LkCa 15, and DM Tau, and simultaneous measurements of $\mathrm{HCO}^{+} J=1 \rightarrow 0$. To derive the disk properties, we fit a standard disk model in which all parameters are power laws of the distance to the star to the data. Possible biases associated with the method are detailed and explained. We compare the properties of the observed disks with similar objects.

Results. We find evidence for a vertical temperature gradient in the disks of MWC 480 and DM Tau, as in AB Aur, but not in LkCa 15. The disk temperatures increase with stellar effective temperature. Except for AB Aur, the bulk of the CO gas is at temperatures smaller than $17 \mathrm{~K}$, below the condensation temperature on grains. We find the scale height of the CO distribution to be larger (by $50 \%$ ) than the expected hydrostatic scale height. The total amount of $\mathrm{CO}$ and the isotopologue ratio depends globally on the star. The more UV luminous objects appear to have more $\mathrm{CO}$, but there is no simple dependency. The $\left[{ }^{13} \mathrm{CO}\right] /\left[\mathrm{HCO}^{+}\right]$ratio is $\sim 600$, with substantial variations between sources, and with radius. The temperature behavior is consistent with expectations, but published chemical models have difficulty reproducing the observed CO quantities. Changes in the slope of the surface density distribution of CO, compared to the continuum emission, suggest a more complex surface density distribution than is usually assumed in models. Vertical mixing seems an important chemical agent, as does photo-dissociation by the ambient UV radiation at the disk's outer edge.
\end{abstract}

Key words. stars: circumstellar matter - planetary systems: protoplanetary disks - stars: individual: LkCa 15 stars: individual: MWC 480 - stars: individual: DM Tau, AB Aur - radio lines: stars

\section{Introduction}

$\mathrm{CO}$ rotation line observations of low-mass and intermediatemass pre-main-sequence (PMS) stars in Taurus-Auriga $(\sim 140$ pc, Kenyon et al. 1994) provide strong evidence that both TTauri and Herbig Ae stars are surrounded by large $\left(R_{\text {out }} \sim 200-800 \mathrm{AU}\right)$ Keplerian disks (GM Aur: Koerner et al. 1993, GG Tau: Dutrey et al. 1994, MWC 480: Mannings et al. 1997). Differences between TTauri and Herbig Ae disks are observed in the very inner disk, very close to the star (Dullemond et al. 2001; Monnier \& Millan-Gabet 2002). However, detailed comparisons, namely based on resolved observations instead of SED analysis, between Herbig Ae disks and TTauri disks are too few to allow conclusions about the whole disk. In particular, there is no specific study on the influence of the spectral type on the disk properties. This can be achieved by comparing the properties of outer disks imaged by millimeter arrays.

The first rotational lines of $\mathrm{CO}(J=1-0$ and $J=2-1)$ mostly trace the cold gas located in the outer disk $(R>30$ AU) since current millimeter arrays are sensitivity limited and do not allow the detection of the warmer gas in the inner disk. In the last years, several detailed studies of CO interferometric maps

${ }^{1}$ Based on observations carried out with the IRAM Plateau de Bure Interferometer. IRAM is supported by INSU/CNRS (France), MPG (Germany), and IGN (Spain). have provided the first quantitative constraints on the physical properties of the outer disks. Among them, the method developed by Dartois et al. (2003) is so far the only way to estimate the overall gas disk structure. Dartois et al. (2003) deduced the vertical temperature gradient of the outer gas disk of DM Tau from a multi-transition, multi-isotope analysis of $\mathrm{CO} J=1-0$ and $J=2-1$ maps. The dust distribution and its temperature can be traced by IR and optical observations and SED modelling (D'Alessio et al. 1999; Dullemond \& Dominik 2004). However Piétu et al. (2006) recently demonstrated that SED analysis is limited by the dust opacity. Due to the high dust opacity in the optical and in the IR, only the disk surface is properly characterized. Images in the mm domain, where the dust is essentially optically thin, revealed in the case of MWC 480 a dust temperature that is significantly lower than those inferred from SED analysis and from CO images. With ALMA, the method described in this paper, which is today only relevant to the outer disk, will become applicable to the inner disk of young stars.

In this paper, we focus on the gas properties deduced from CO data. The analysis of the millimeter continuum data, observed in the meantime, is presented separately (Piétu et al. 2006). Here, we generalize the method developed by Dartois et al. (2003) and we apply it to two Keplerian disks surrounding the TTauri star LkCa 15 (spec. type K5, Duvert et al. 2000) 
Table 1. Star properties.

\begin{tabular}{lllllll}
\hline \hline Source & Right ascension & Declination & Spec. type & Effective temp. $(\mathrm{K})$ & Stellar lum. $\left(L_{\odot}\right)$ & CO paper \\
\hline LkCa 15 & $04: 39: 17.790$ & $22: 21: 03.34$ & K5 & 4350 & 0.74 & 1,2 \\
MWC 480 & $04: 58: 46.264$ & $29: 50: 36.86$ & A4 & 8460 & 11.5 & 1,2 \\
DM Tau & $04: 33: 48.733$ & $18: 10: 09.89$ & M1 & 3720 & 0.25 & 3 \\
AB Aur & $04: 55: 45.843$ & $30: 33: 04.21$ & A0/A1 & 10000 & 52.5 & 5 \\
HD 34282 & $05: 16: 00.491$ & $-09: 48: 35.45$ & A1/A0 & 9440 & 29 & 4 \\
GM Aur & $04: 55: 10.98$ & $30: 21: 59.5$ & K7 & 4060 & 0.74 & 6 \\
\hline
\end{tabular}

Columns 2 and 3: J2000 coordinates deduced from the fit of the $1.3 \mathrm{~mm}$ continuum map of the PdBI. Errors bars on the astrometry are of order $\leq 0.05^{\prime \prime}$. Columns 4,5 , and 6 , the spectral type, effective temperature and the stellar luminosity are those given in Simon et al. (2000) and Piétu et al. (2003) for HD 34282 and van den Ancker et al. (1998) for AB Aur. Column 6, CO interferometric papers are: $1=$ this paper, $2=$ Simon et al. (2000), 3 = Dartois et al. (2003), 4 = Piétu et al. (2003), 5 = Piétu et al. (2005), and 6 = Dutrey et al. (1998).

and the Herbig Ae star MWC 480 (spec. type A4, Simon et al. 2000). By improving the method, we also discuss the estimate of disk scale heights. Then, we compare their properties with those surrounding Herbig Ae and TTauri disks such as AB Aur (Piétu et al. 2005), HD 34282 (Piétu et al. 2003), and DM Tau (Dartois et al. 2003). After presenting the sample of stars and the observations in Sect. 2, we describe the improved analysis method in Sect. 3. Results are presented in Sect. 4 and their implications discussed in Sect. 5.

\section{Sample of tars and observations}

\subsection{Sample of stars}

The stars were selected to sample the wider spectral type range with only a few objects. Table 1 gives the coordinates and spectral type of the observed stars, as well as of HD 34282 and GM Aur, which were observed in ${ }^{12} \mathrm{CO}$ by Piétu et al. (2003) and Dutrey et al. (1998) respectively. Our stars have spectral types ranging from $\mathrm{M} 1$ to $\mathrm{A} 0$. All of them have disks observed at several wavelengths and are located in a hole or at the edge of their parent $\mathrm{CO}$ cloud.

DM Tau: DM Tau is a bona fide T Tauri star. Guilloteau \& Dutrey (1994) first detected CO lines indicating Keplerian rotation using the IRAM $30 \mathrm{~m}$ telescope. Guilloteau \& Dutrey (1998) have confirmed those findings by resolving the emission of the ${ }^{12} \mathrm{CO} J=1 \rightarrow 0$ emission using IRAM Plateau de Bure interferometer (PdBI) and derived physical parameters of its protoplanetary disk and mass for the central star. Dartois et al. (2003) performed the first high resolution multi-transition, multi-isotope study of $\mathrm{CO}$, and demonstrated the existence of a vertical temperature gradient in the disk.

LkCa 15: this is a CTT isolated from its parent cloud. Duvert et al. (2000) and Qi et al. (2003) observed the disk in ${ }^{12} \mathrm{CO}$ and in $\mathrm{HCO}^{+}$. High resolution ${ }^{12} \mathrm{CO}$ data was obtained by Simon et al. (2000), who used it to derive the stellar mass.

MWC 480: the existence of a disk around this A4 Herbig Ae star was first reported by Mannings et al. (1997). Using data from OVRO, they perform a modelling of the gas disk consistent with Keplerian rotation. More recently, Simon et al. (2000) confirmed that the rotation was Keplerian and measured the stellar mass from the $\mathrm{CO}$ pattern.

AB Aurigae: $A B$ Aur is considered as the proto-type of the Herbig Ae star (of spectral type A0), which is surrounded by a large $\mathrm{CO}$ and dust disk. The reflection nebula, observed in the optical, extends far away from the star (Grady et al. 1999). Semenov et al. (2005) used the 30-m to observe the chemistry of the envelope. Piétu et al. (2005) has analyzed the disk using high angular resolution images of $\mathrm{CO}$ and isotopologues from the IRAM array and found that, surprisingly, the rotation is not Keplerian. A central hole was detected in the continuum images and in the more optically thin ${ }^{13} \mathrm{CO}$ and $\mathrm{C}^{18} \mathrm{O}$ lines.

\section{2. $P d B I$ data}

For DM Tau, LkCa 15, and MWC 480, the ${ }^{12} \mathrm{CO} J=2-1$ data were observed in snapshot mode during the winter of 1997/1998 in D, C2 and B1 configurations. Details about the data quality and reduction are given in Simon et al. (2000) and in Dutrey et al. (1998). The ${ }^{12} \mathrm{CO} J=2-1$ data were smoothed to $0.2 \mathrm{~km} \mathrm{~s}^{-1}$ spectral resolution. The $\mathrm{HCO}^{+} J=1 \rightarrow 0$ data were obtained simultaneously: a $20 \mathrm{MHz} / 512$ channels correlator unit provided a spectral resolution of $0.13 \mathrm{~km} \mathrm{~s}^{-1}$. The ${ }^{13} \mathrm{CO} J=1-0$ and $J=2-1$ observations of DM Tau are described in Dartois et al. (2003).

We obtained complementary ${ }^{13} \mathrm{CO} J=2-1$ and $J=1-0$ (together with $\mathrm{C}^{18} \mathrm{O} J=1-0$ observations) of MWC 480 and LkCa 15. These observations were carried out in snapshot mode (partly sharing time with $\mathrm{AB}$ Aur) in winter periods between 2002 and 2004. Configurations D, C2, and B1 were used at 5 antennas. MWC 480 has also shortly been observed in A configuration simultaneously with $\mathrm{AB}$ Aur. Finally, data was obtained in the largest $\mathrm{A}^{+}$configuration for $\mathrm{LkCa} 15$ and MWC 480 in January 2006 (for a description of these observations, see Piétu et al. 2006). Both at $1.3 \mathrm{~mm}$ and $2.7 \mathrm{~mm}$, the tuning was doubleside-band (DSB). The backend was a correlator with one band of $20 \mathrm{MHz}, 512$ channels (spectral resolution $0.11 \mathrm{~km} \mathrm{~s}^{-1}$ ) centered on the ${ }^{13} \mathrm{CO} J=1-0$, one on the $\mathrm{C}^{18} \mathrm{O} J=1-0$ line, and a third one centered on the ${ }^{13} \mathrm{CO} J=2 \rightarrow 1$ line (spectral resolution $0.05 \mathrm{~km} \mathrm{~s}^{-1}$ ), and 2 bands of $160 \mathrm{MHz}$ for each of the $1.3 \mathrm{~mm}$ and $2.7 \mathrm{~mm}$ continuum. The phase and secondary flux calibrators were $0415+379$ and $0528+134$. The rms phase noise was $8^{\circ}$ to $25^{\circ}$ and $15^{\circ}$ to $50^{\circ}$ at $3.4 \mathrm{~mm}$ and $1.3 \mathrm{~mm}$, respectively (up to $70^{\circ}$ on the $700 \mathrm{~m}$ baselines), which introduced position errors of $\leq 0.05^{\prime \prime}$. The estimated seeing is about $0.2^{\prime \prime}$.

Flux density calibration is a critical step in such projects. The kinetic temperature, which is deduced from the modelling, is directly proportional to the measured flux density. This is even more problematic since we compare data obtained over several years. Hence, we took great care of the relative flux calibration by using not only MWC 349 as a primary flux reference, assuming a flux $S(v)=0.95(v / 87 \mathrm{GHz})^{0.60}$ (see the IRAM flux report 14) but also by always having our own internal reference in the 


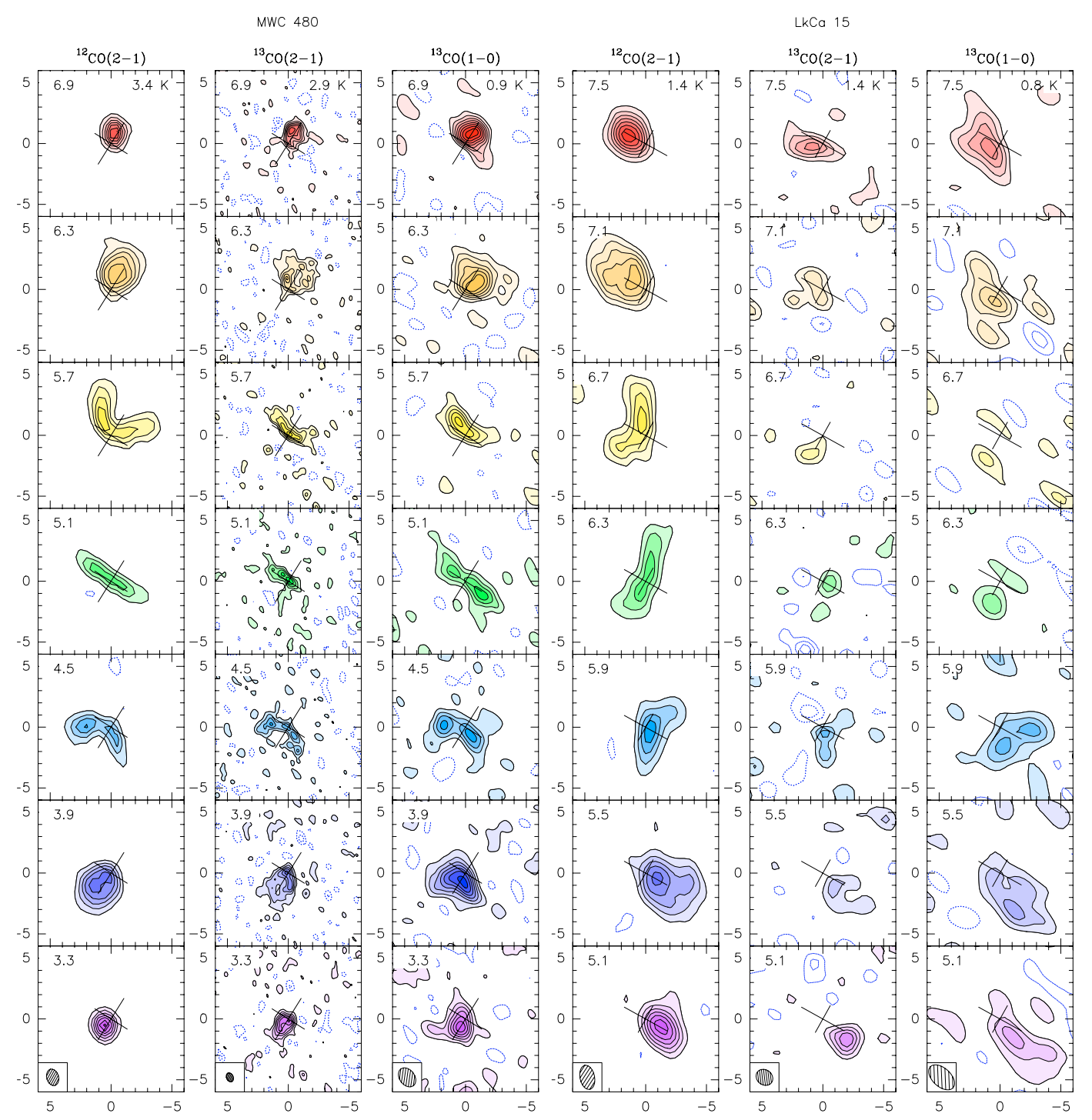

Fig. 1. Channel maps of the ${ }^{12} \mathrm{CO}$ and ${ }^{13} \mathrm{CO}$ line emission towards MWC 480 and $\mathrm{LkCa} 15$. The synthesized beam is indicated in each panel. The cross indicates the direction of the major and minor axes of each disk, as respectively derived from the analysis of the line data. The velocity of the channel is indicated in the upper left corner of each panel (LSR velocity in $\mathrm{km} \mathrm{s}^{-1}$ ). A $200 \mathrm{~m}$ taper has been applied on the ${ }^{13} \mathrm{CO} J=2 \rightarrow 1$ data of $\mathrm{LkCa} 15$, which were obtained at higher angular resolution. Left: MWC 480 Left: ${ }^{12} \mathrm{CO} J=2 \rightarrow 1$ line, spatial resolution $1.38 \times 0.99^{\prime \prime}$ at PA $16^{\circ}$, contour spacing $200 \mathrm{mJy} /$ beam, or $3.4 \mathrm{~K}$, or $3.6 \sigma$. Middle: ${ }^{13} \mathrm{CO} J=2 \rightarrow 1$ line, spatial resolution $0.77 \times 0.57$ at PA $26^{\circ}$, contour spacing $50 \mathrm{mJy} /$ beam, or $2.9 \mathrm{~K}$, or $1.9 \sigma$. Right: ${ }^{13} \mathrm{CO} J=1 \rightarrow 0$ line, spatial resolution $1.72 \times 1.24^{\prime \prime}$ at PA $37^{\circ}$, contour spacing $20 \mathrm{mJy} / \mathrm{beam}$, or $0.9 \mathrm{~K}$, or $1.8 \sigma$. Negative contours are dashed, and the zero contour is omitted. Right: LkCa 15 Left: ${ }^{12} \mathrm{CO} J=2 \rightarrow 1$ line, spatial resolution $2.01 \times 1.20^{\prime \prime}$ at PA $12^{\circ}$, contour spacing $150 \mathrm{mJy} /$ beam, or $1.4 \mathrm{~K}$, or $2.5 \sigma$. Middle: ${ }^{13} \mathrm{CO} J=2 \rightarrow 1$ line, spatial resolution $1.46 \times 1.24$ at PA $52^{\circ}$, contour spacing $100 \mathrm{mJy} /$ beam, or $1.4 \mathrm{~K}$, or $1.6 \sigma$. Right: ${ }^{13} \mathrm{CO} J=1 \rightarrow 0$ line, spatial resolution $2.55 \times 1.51^{\prime \prime}$ at PA $47^{\circ}$, contour spacing $30 \mathrm{mJy} / \mathrm{beam}$, or $0.8 \mathrm{~K}$, or $1.8 \sigma$. Negative contours are dashed, and the zero contour is omitted.

observations. For this purpose we applied two methods: i) we used MWC 480 as a reference because its continuum emission is reasonably compact and bright, ii) we also checked the coherency of the flux calibration on the spectral index of DM Tau, as described in Dartois et al. (2003). We cross-checked all methods and in this way obtained a reliable relative flux calibration from one frequency to another.

\subsection{Data reduction and results}

We used the GILDAS ${ }^{1}$ software package (Pety 2005) to reduce the data and as a framework to implement our

${ }^{1}$ See http://www.iram. fr/IRAMFR/GILDAS for more information about the GILDAS software. minimization technique. Figure 1 presents some channel maps of ${ }^{12} \mathrm{CO},{ }^{13} \mathrm{CO} J=2 \rightarrow 1$, and ${ }^{13} \mathrm{CO} J=1 \rightarrow 0$ for $\mathrm{LkCa} 15$ and MWC 480. Similar figures for DM Tau can be found in Dartois et al. (2003), and for AB Aur in Piétu et al. (2005). A velocity smoothing to $0.6 \mathrm{~km} \mathrm{~s}^{-1}$ (for MWC 480) and $0.4 \mathrm{~km} \mathrm{~s}^{-1}$ (for LkCa 15) was applied to produce the images displayed in Fig. 1. However, in the analysis done by minimization, we used spectral resolutions of $0.20 \mathrm{~km} \mathrm{~s}^{-1}\left({ }^{12} \mathrm{CO}\right.$ and $\left.\mathrm{HCO}^{+}\right)$and $0.21 \mathrm{~km} \mathrm{~s}^{-1}$ $\left({ }^{13} \mathrm{CO}\right)$, and natural weighting was retained. The $\mathrm{HCO}^{+}$images are presented in Fig. 2. The typical angular resolution of these images is 3-4". For LkCa 15, the line flux is compatible with the result quoted by Duvert et al. (2000) and Qi et al. (2003), and for DM Tau with the single-dish measurement of Dutrey et al. (1997). 


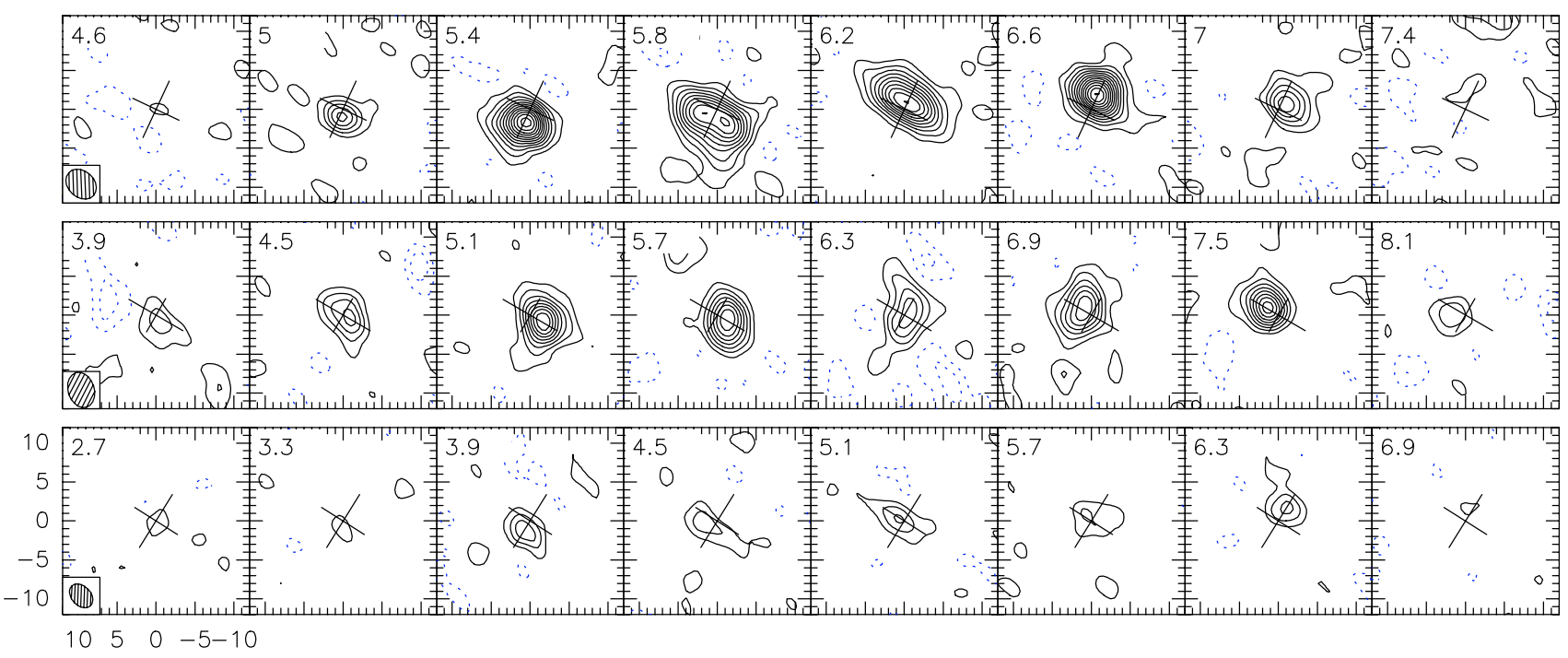

Fig. 2. Channel maps of the $\mathrm{HCO}^{+} J=1 \rightarrow 0$ line emission towards DM Tau, LkCa 15, and MWC 480. The cross indicates the direction of the major and minor axes of each disk. The velocity of the channel is indicated in the upper left corner of each panel (LSR velocity in km s ${ }^{-1}$ ). For displaying purpose, the spectral resolution is $0.6 \mathrm{~km} \mathrm{~s}^{-1}$ for MWC 480 and LkCa 15, and $0.4 \mathrm{~km} \mathrm{~s}^{-1}$ for DM Tau. All contour steps are $20 \mathrm{mJy} /$ beam $(\sim 2 \sigma)$, negative contours are dashed, and the zero contour is omitted. Top: DM Tau, spatial resolution is $4.3 \times 3.3^{\prime \prime}$ at PA $47^{\circ}$, contour step $0.22 \mathrm{~K}$. Middle: LkCa 15, spatial resolution is $4.6 \times 3.4^{\prime \prime}$ at PA $19^{\circ}$, contour step $0.25 \mathrm{~K}$. Bottom: MWC 480, spatial resolution is $3.5 \times 2.5^{\prime \prime}$ at PA $42^{\circ}$, contour step $0.36 \mathrm{~K}$.

\section{Model description and method of analysis}

In this section, we summarize the model properties and describe the improvements since the first version (Dutrey et al. 1994).

\subsection{Solving the radiative transfer equation}

To solve the radiative transfer equation coupled to the statistical equilibrium, we have written a new code, offering non LTE capabilities. More details are given in Pavlyuchenkov et al. (2007). The LTE part, which is sufficient for a low energy level of rotational CO lines ( $J=1-0$ and $J=2-1)$, is strictly similar to that of Dutrey et al. (1994), and uses ray-tracing to compute the images. The $\mathrm{HCO}^{+}$data was also analyzed in LTE mode (see Sect. 3.2.3 for the interpretation of this assumption). We use a scheme of nested grids with regular sampling to provide sufficient resolution in the inner parts of the disk while avoiding excessive computing time.

\subsection{Description of the physical parameters}

For each spectral line, the relevant physical quantities that control the line emission are assumed to vary like a power law as a function of radius (see Sect. 3.2.1 for the interpretation of this term), and, except for the density, do not depend on height above the disk plane. The exponent is taken as positive if the quantity decreases with radius:

$a(r)=a_{0}\left(r / R_{a}\right)^{-e_{a}}$

For each molecular line, the disk is thus described by the following parameters:

- $X_{0}, Y_{0}$, the star position, and $V_{\text {disk }}$, the systemic velocity;

- PA, the position angle of the disk axis, and $i$ the inclination;

- $V_{0}$, the rotation velocity at a reference radius $R_{v}$, and $v$ the exponent of the velocity law. With our convention, $v=0.5$ corresponds to a Keplerian rotation. Furthermore, the disk is oriented so that the $V_{0}$ is always positive. Accordingly, PA varies between $0^{\circ}$ and $360^{\circ}$, while $i$ is constrained between $-90^{\circ}$ and $90^{\circ}$ (see Fig. 3);

- $T_{\mathrm{m}}$ and $q_{m}$, the temperature value at a reference radius $R_{T}$ and its exponent (see Sect. 3.2.3);

$-\mathrm{d} V$, the local line width, and its exponent $e_{v}$. The interpretation of $\mathrm{d} V$ is discussed in Sect. 3.2.6;

- $\Sigma_{m}$, the molecular surface density at a radius $R_{\Sigma}$ and its exponent $p_{m}$;

- $R_{\text {out }}$, the outer radius of the emission, and $R_{\text {in }}$, the inner radius;

- $h_{m}$, the scale height of the molecular distribution at a radius $R_{h}$, and its exponent $e_{h}$ : it is assumed that the density distribution is Gaussian, with

$n(r, z)=\frac{\Sigma(r)}{h(r) \sqrt{\pi}} \exp \left[-(z / h(r))^{2}\right]$

(note that with this definition, $e_{h}<0$ in realistic disks).

thus giving a grand total of 17 parameters to describe the emission.

It is important to realize that all these parameters can actually be constrained for the lines we have observed, under the above assumption of power laws. This comes from two specific properties of proto-planetary disks: i) the rapid decrease of the surface density with radius, and ii) the known kinematic pattern. The only exception is the inner radius, $R_{\text {in }}$, for which an upper limit of typically 20-30 AU can only be obtained.

The use of power laws is appropriate for the velocity, provided the disk self-gravity remains small. Power laws have been shown to be a good approximation for the kinetic temperature distribution (see, e.g., Chiang \& Goldreich 1997), and thus to the scale height prescription. For the surface density, power laws are often justified for the mass distribution based on the $\alpha$ prescription of the viscosity with a constant accretion rate: selfsimilar solutions to the disk evolution imply power laws with an exponential edge (Hartmann et al. 1998). Although Malbet et al. (2001) pointed out some limitations of this assumption for the inner parts $(r<30 \mathrm{AU})$ of the disk, the approximation remains reasonable beyond 50 AU. However, although this may 


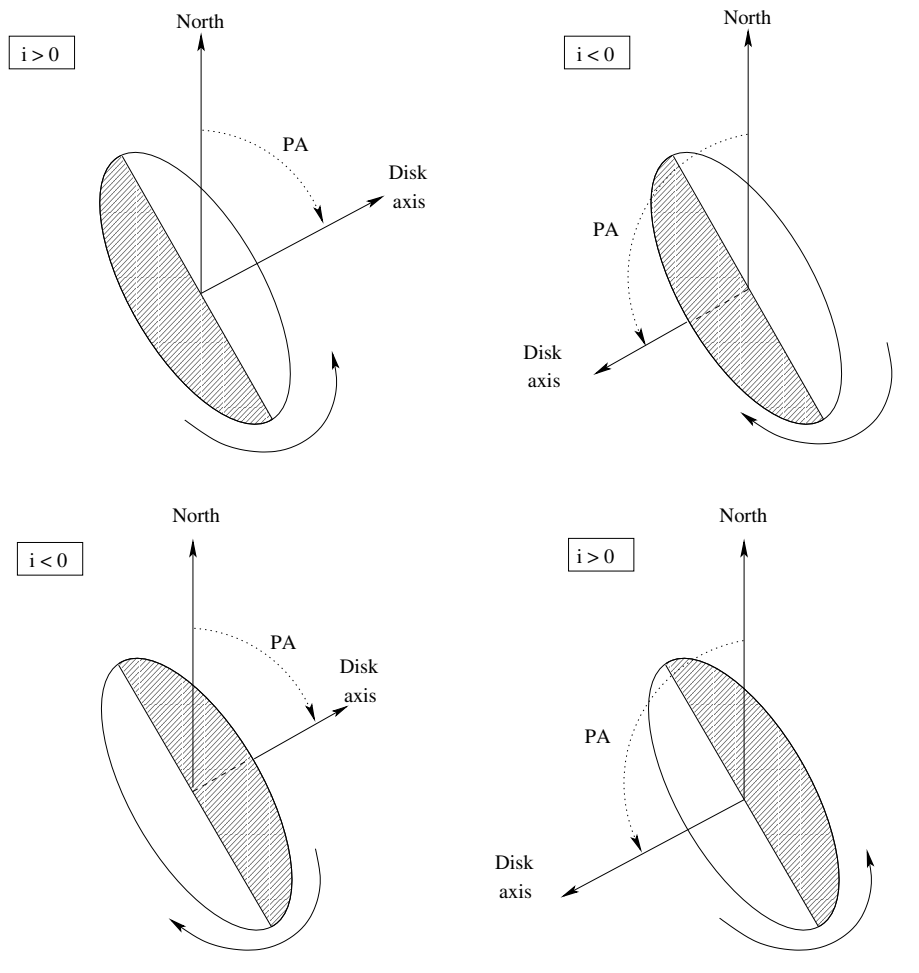

Fig. 3. Geometrical convention: four disk configurations yielding the same aspect ratio when projected are presented. The shaded area corresponds to the part of the disk that is closer to us than the plane of the sky containing the disk axis. The curved arrow represents the sense of rotation (this defines the disk axis). The projected velocities allow us to distinguish between one of the two columns (for the two cases presented on the left, the red-shifted part would be the lower part, and the contrary for the two cases on the right). It is possible to determine the sign of the inclination either with the asymmetry at the systemic velocity, if a sufficient signal-to-noise ratio is available, or by other means, e.g., scattered images in the optical and near-IR. PA is classically designed to be positive East from North.

be valid for $\mathrm{H}_{2}$, chemical effects may lead to significant differences for any molecule. Given the limited spatial dynamic range and signal-to-noise ratio provided by current (sub-)millimeter arrays, using more sophisticated prescriptions is not yet justified, and power laws offer a first-order approximation of the overall molecular distribution.

\subsubsection{Spherical vs. cylindrical representation}

To describe the disk and its parameters, we can use either cylindrical or spherical coordinates. The description given in the previous sub-section is unambiguous for thin disks (where $h(r) \ll$ $r$ ), However, for molecular disks with outer radii as large as $800 \mathrm{AU}$, the disk thickness is no longer small, and the classical theory of hydrostatic scale height, which uses cylindrical coordinates, is only valid in the small $h / r$ limit. It may then become significant to distinguish between a spherical representation, where the functions depends on the distance to the star $(r=d)$ and the height above the disk plane, $z$

$F=f(d, z)$

and a cylindrical representation, where the functions depends on the projected distance on the disk $(r=\rho)$ and height $z$

$F=f(\rho, z)$,
Table 2. Cylindrical vs. spherical description.

\begin{tabular}{lccc}
\hline \hline Line & ${ }^{13} \mathrm{CO}(1-0)$ & ${ }^{13} \mathrm{CO}(2-1)$ & ${ }^{12} \mathrm{CO}(2-1)$ \\
\hline$R_{\text {out }}(\mathrm{AU})$ & $791 \pm 33$ & $725 \pm 24$ & $868 \pm 15$ \\
$T_{0}(\mathrm{~K})$ & $9.1 \pm 0.3$ & $14.7 \pm 0.3$ & $24.2 \pm 0.2$ \\
$q$ & $-0.17 \pm 0.09$ & $0.18 \pm 0.05$ & $0.46 \pm 0.01$ \\
$V_{0}\left(\mathrm{~km} \mathrm{~s}^{-1}\right)$ & $2.12 \pm 0.07$ & $2.02 \pm 0.06$ & $2.09 \pm 0.06$ \\
$v$ & 0.5 & 0.5 & 0.5 \\
$\mathrm{~d} V\left(\mathrm{~km} \mathrm{~s}^{-1}\right)$ & $0.133 \pm 0.015$ & $0.136 \pm 0.015$ & $0.147 \pm 0.010$ \\
$e_{v}$ & $-0.28 \pm 0.08$ & $-0.04 \pm 0.07$ & $0.08 \pm 0.04$ \\
$\Sigma_{m}\left(\mathrm{~cm}^{-2}\right)$ & $26.9 \pm 1.7 \times 10^{16}$ & $41.4 \pm 6.1 \times 10^{16}$ & $37.4 \pm 9.1 \times 10^{18}$ \\
$p_{m}$ & $3.3 \pm 0.3$ & $3.7 \pm 0.4$ & $4.5 \pm 0.5$ \\
$h_{m}(\mathrm{AU})$ & $27.1 \pm 4.4$ & $27.8 \pm 2.9$ & $33.4 \pm 2.3$ \\
$e_{h}$ & $-1.07 \pm 0.20$ & $-1.09 \pm 0.10$ & $-0.99 \pm 0.04$ \\
\hline$\chi^{2}$ & 513329.3 & 514557.2 & 136376.5 \\
\hline \multicolumn{4}{c}{$\mathrm{Spherical}$} \\
\hline$R_{\text {out }}$ & $804 \pm 34$ & $737 \pm 20$ \\
$T_{0}$ & $9.3 \pm 0.3$ & $15.2 \pm 0.4$ & $898 \pm 12$ \\
$q$ & $-0.14 \pm 0.09$ & $0.20 \pm 0.05$ & $26.7 \pm 0.2$ \\
$V_{0}\left(\mathrm{~km} \mathrm{~s}^{-1}\right)$ & $2.16 \pm 0.07$ & $2.07 \pm 0.06$ & $2.08 \pm 0.01$ \\
$v$ & 0.5 & 0.5 & 0.5 \\
$\mathrm{~d} V\left(\mathrm{~km} \mathrm{~s}^{-1}\right)$ & $0.135 \pm 0.016$ & $0.137 \pm 0.015$ & $0.183 \pm 0.012$ \\
$e_{v}$ & $-0.26 \pm 0.08$ & $-0.05 \pm 0.06$ & $0.17 \pm 0.05$ \\
$\Sigma_{m}\left(\mathrm{~cm}^{-2}\right)$ & $25.7 \pm 1.8 \times 10^{16}$ & $35.1 \pm 5.3 \times 10^{16}$ & $41.1 \pm 7.4 \times 10^{17}$ \\
$p_{m}$ & $3.2 \pm 0.3$ & $3.6 \pm 0.4$ & $3.4 \pm 0.4$ \\
$h_{m}(\mathrm{AU})$ & $20.7 \pm 2.2$ & $23.4 \pm 2.4$ & $19.7 \pm 1.4$ \\
$e_{h}$ & $-1.32 \pm 0.18$ & $-1.13 \pm 0.12$ & $-1.25 \pm 0.06$ \\
\hline$\chi^{2}$ & 513329.5 & 514558.9 & 136451.5 \\
\hline
\end{tabular}

Results for DM Tau with "free" scale-height and "free" line-width (see Sects. 3.2.5 and 3.2.6 for more details) in the cylindrical and spherical representations. Caution: this table is here to illustrate the dependence on the geometry of as many parameters as possible. The best parameters to be applied to DM Tau should be taken from Table 3.

with $d^{2}=\rho^{2}+z^{2}$. It is obvious that the exponent of the power laws will depend slightly on which description is selected. We have evaluated the differences between the two different representations (see Table 2): as expected, the results are very similar for ${ }^{13} \mathrm{CO}$, but the cylindrical representation is $\sim 8$ sigma better for ${ }^{12} \mathrm{CO} J=2 \rightarrow 1$ (the most affected transition because of its higher opacity). The most affected parameters are $\Sigma$ and $h$. However, with the exception of the scale height, the effects remain small compared to the uncertainties. Accordingly, we have arbitrarily decided to represent all parameters in terms of cylindrical laws, i.e., the radius is $r=\rho$ in the model description.

\subsubsection{Reference radius}

Unless $p_{m} \simeq 0$, the choice of the reference radius $R_{\Sigma}$ will affect the relative error bar on $\Sigma_{m}, \delta \Sigma_{m} / \Sigma_{m}$. Depending on the angular resolution and on the overall extent of the emission, for each molecular line, there is a different optimal radius $R_{\Sigma}$ that minimizes this relative error. The same is true for all other reference radii for the power laws. This makes direct comparison at an arbitrary radius not straightforward. To circumvent this problem, we have determined $\Sigma_{m}, \delta \Sigma_{m}$ as a function of the reference radius for each transition, and selected the $R_{\Sigma}$ giving the best $\mathrm{S} / \mathrm{N}$ ratio. Results are given in Figs. 4-6: note that the curves $\Sigma_{m}\left(R_{\Sigma}\right)$ should not be interpreted as independent estimates of the local surface density at different radii: they explicitly rely on the assumption of a single power law throughout the whole disk. 

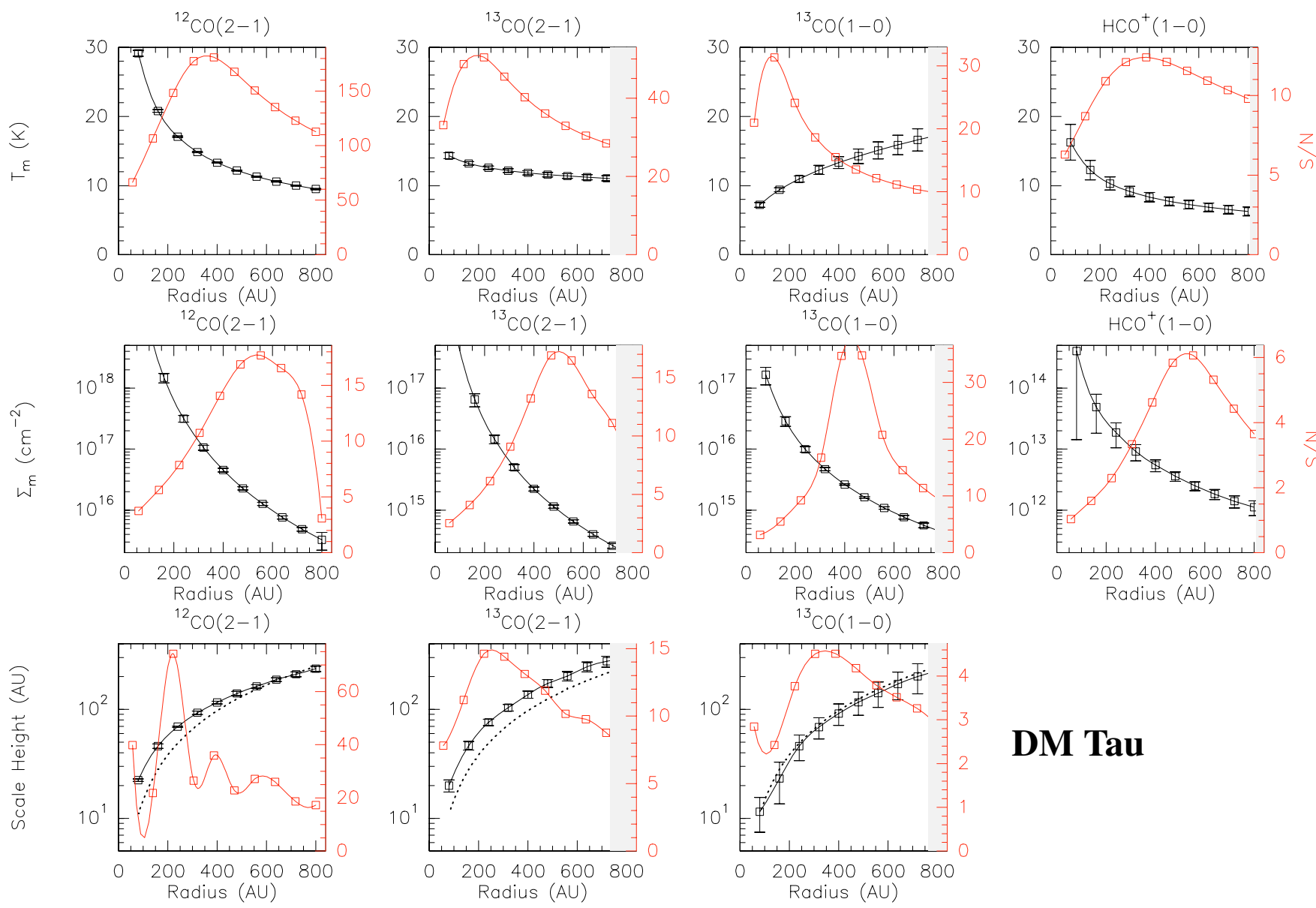

\section{DM Tau}

Fig. 4. From left to right ${ }^{12} \mathrm{CO} J=2 \rightarrow 1,{ }^{13} \mathrm{CO} J=2 \rightarrow 1,{ }^{13} \mathrm{CO} J=1 \rightarrow 0$ and $\mathrm{HCO}^{+} J=1 \rightarrow 0$ in DM Tau. Top: temperature $T_{m}$ (black curve, left axis) and signal-to-noise ratio for the temperature $T_{m} / \delta T_{m}$ (grey curve, right axis) as a function of reference radius $R_{T}$. Middle: surface density $\Sigma_{m}$ (left axis) and signal-to-noise ratio for the surface density $\Sigma_{m} / \delta \Sigma_{m}$ (right axis) as a function of reference radius $R_{\Sigma}$. Bottom: scale height $h$ (left axis) and signal-to-noise ratio for the scale height $h / \delta h$ (right axis) as a function of reference radius $R_{h}$.

The same arguments can be applied to all other power laws, in particular to the scale height and the temperature. However, for the latter, the exponent is usually small $(q=-0.2-0.5)$, so the choice of the reference radius is less critical. Figure 4 shows the variation of the parameters (here $\Sigma_{m}, T_{m}$, and $h$ ) and of the signal-to-noise ratio as a function of the reference radius for DM Tau. This is a reanalysis of the data published by Dartois et al. (2003). This method gives results that are consistent with previous work, but allows a more precise determination of the parameters (especially the surface density as stated above), by determining in which region they are constrained. Figure 4 indicates that the temperature is determined around 100-200 AU, while the surface densities are constrained in the 300-500 AU region. Somewhat smaller values apply to the other sources, LkCa 15 and MWC 480 (see Figs. 5-6).

\subsubsection{Temperature law}

The interpretation of $T_{m}$ (and as a consequence of $\Sigma_{m}$ ) depends on the specific model of radiative transfer being used. In this paper, we are dealing with CO isotopologues, for which LTE is a good approximation: $T_{m}$ is then the kinetic temperature. For transitions which may not be thermalized, two different radiative transfer models may be used. We can apply an LTE approximation: since we are fitting brightness distributions, $T_{m}$ is in this case the excitation temperature $T_{\mathrm{ex}}$, and the surface density $\Sigma_{m}$ is computed assuming the same temperature controls all level population, i.e., the partition function. We can also solve for molecular line excitation using a non-LTE statistical equilibrium code: $T_{m}$ is then the kinetic temperature, and $\Sigma_{m}$ the total molecule surface density, within the limitations of the radiative transfer code accuracy. In this paper, only the LTE mode was used.

\subsubsection{Surface density}

The surface density is expected to fall off rapidly with distance from the $\operatorname{star}\left(p\left(\mathrm{H}_{2}\right) \simeq 1-2\right)$, and in particular much more rapidly than the temperature $(q \simeq 0.5)$. Remembering that, because of the partition function, the line opacity of a $J=1-0$ transition scales as $\Sigma_{m} / T^{2}$ at high enough temperatures (for constant line width), this indicates that, in general, the central part of the disk is much more optically thick than the outer regions. For the detectable lines, the temperature can be derived from the emission from this optically thick core, while the surface density $\Sigma_{m}$ is derived from the optically thin region. This remains valid provided the same temperature law applies to the two regions. For the weakest lines, the optically thick core may be too small, and the temperature exponent remains essentially unconstrained: this should be reflected to by the error bars on the temperature. In such cases, however, the line emission scales as $\Sigma_{m} / T$ (for $J=1-0$ line). 

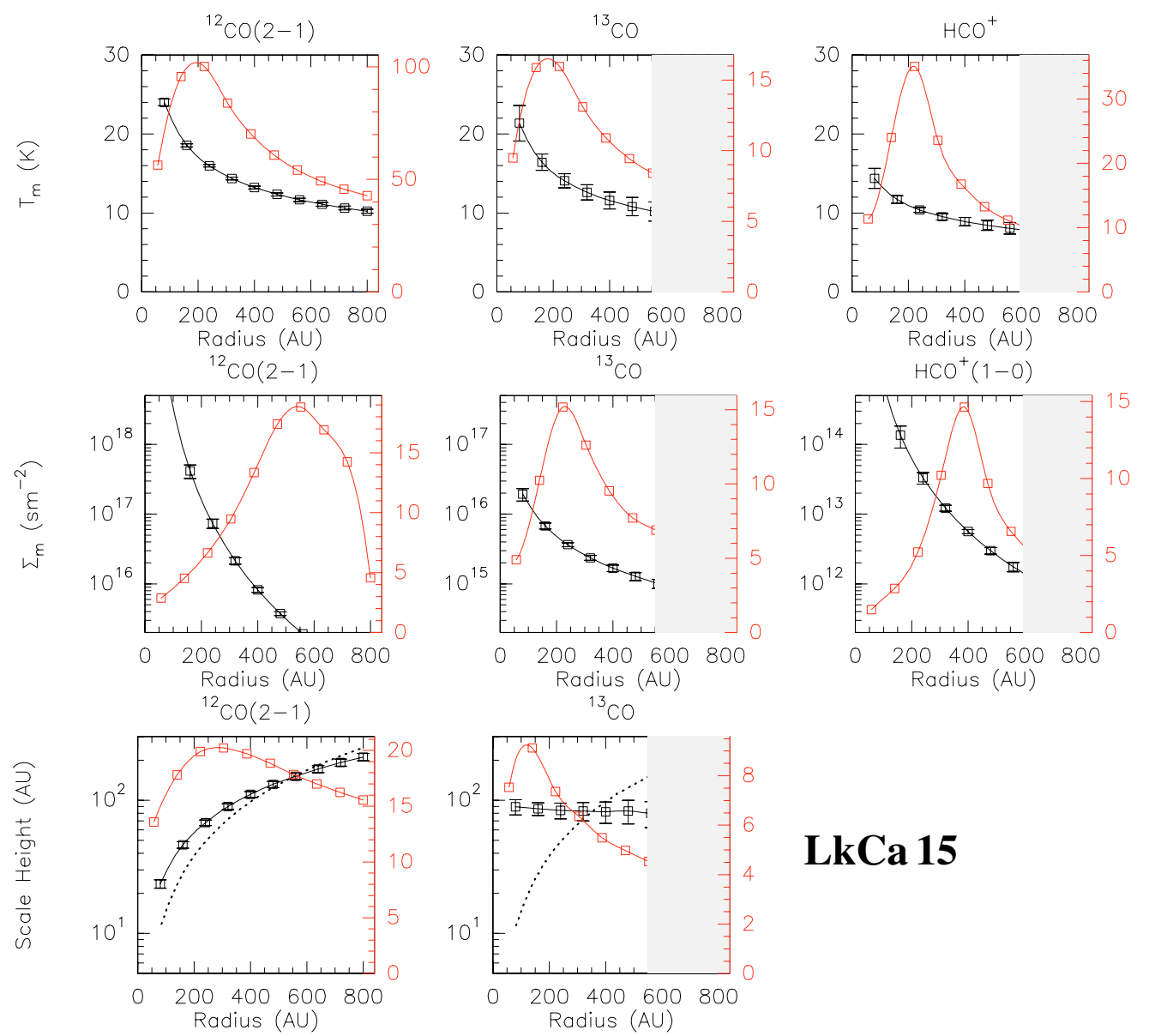

\section{LkCa 15}

Fig. 5. From left to right ${ }^{12} \mathrm{CO},{ }^{13} \mathrm{CO}$ and $\mathrm{HCO}^{+} \quad J=1 \rightarrow 0$ in LkCa 15. Top: temperature $T_{m}$ (black curve, left axis) and signal-to-noise ratio for the temperature $T_{m} / \delta T_{m}$ (grey curve, right axis) as a function of reference radius $R_{T}$. Middle: surface density $\Sigma_{m}$ (left axis) and signal-to-noise ratio for the surface density $\Sigma_{m} / \delta \Sigma_{m}$ (right axis) as a function of reference radius $R_{\Sigma}$. Bottom: scale height $h$ (left axis) and signal-to-noise ratio for the scale height $h / \delta h$ (right axis) as a function of reference radius $R_{h}$. For $\mathrm{HCO}^{+}$the temperature and surface density were adjusted separately: the curves should be used as an indicator of the region over which these values are actually constrained, but not as a quantitative measure in terms of $\mathrm{S} / \mathrm{N}$, as the coupling between temperature and surface density is ignored.

If the molecule is very abundant, such as $\mathrm{CO}$, the local line core may be optically thick almost throughout the disk. However, the local line wings remain optically thin: if the velocity resolution is high enough, this information can be used to constrain the opacity, and hence the surface density, although less accurately. To illustrate this point, let us consider the case of a face-on disk. The apparent width of the line profile is then controlled only by the internal velocity dispersion (turbulent + thermal) and by the line opacity. In the outer parts, the line is optically thin and of Gaussian shape, while in the inner parts, the line is broadened by the large opacity, and the profile tends towards a square shape, with width proportional to $\sqrt{\ln (\tau)}$. The evolution of the line profiles as a function of radius indicates at which radius $\tau=1$, and thus constrains the molecular surface density. Note that if the spectral resolution is insufficient to properly sample the line width, this is no longer possible. Current correlators are not instrumentally limited, but smoothing can be necessary when the sensitivity is the limiting factor. If the disk is not seen face on, the systematic velocity gradient get superimposed on this local line broadening, but does not change the fundamental relationship between the apparent (local) line width and the opacity. In any case, since the effect goes as $\sqrt{\ln (\tau)}$, measuring the surface density by such a method is expected to be rather unprecise, and will result in larger errors. Furthermore, it should be noted that this opacity broadening effect results in a slight coupling of the line width parameters, $\mathrm{d} V$ and $e_{v}$, to the surface density.

\subsubsection{Scale height}

The existence of a known kinematic pattern also allows us to constrain the scale height parameters $h_{0}$ and $e_{h}$. Unless the disk is seen face on, for an optically thin line, the velocity range intersected along a line of sight will depend on the disk inclination and flaring, resulting in a coupling between scale height and line width. For an optically thick line, the difference in inclinations between the front and back regions of the disk can also be measured. The spatial distribution of the emission as a function of projected velocity (or function of the velocity channels) along the line of sight will depend on the scale height of the disk (this is illustrated for the opacity by Fig. 3 from Dartois et al. 2003). At first glance, the expected scale heights, of order $50 \mathrm{AU}$ at $300 \mathrm{AU}$ from the star, would appear too small compared to the resolution of $0.7-1.5^{\prime \prime}$ (100 to $200 \mathrm{AU}$ ), and the exponent $e_{h}$ even more difficult to constrain. However, millimeter interferometry, being a heterodyne technique, allows phase referencing between velocity channels, and the precision in relative positions is equal to the spatial resolution divided by the signal-to-noise ratio (as our bandpass accuracy, about a degree, is not the limiting 

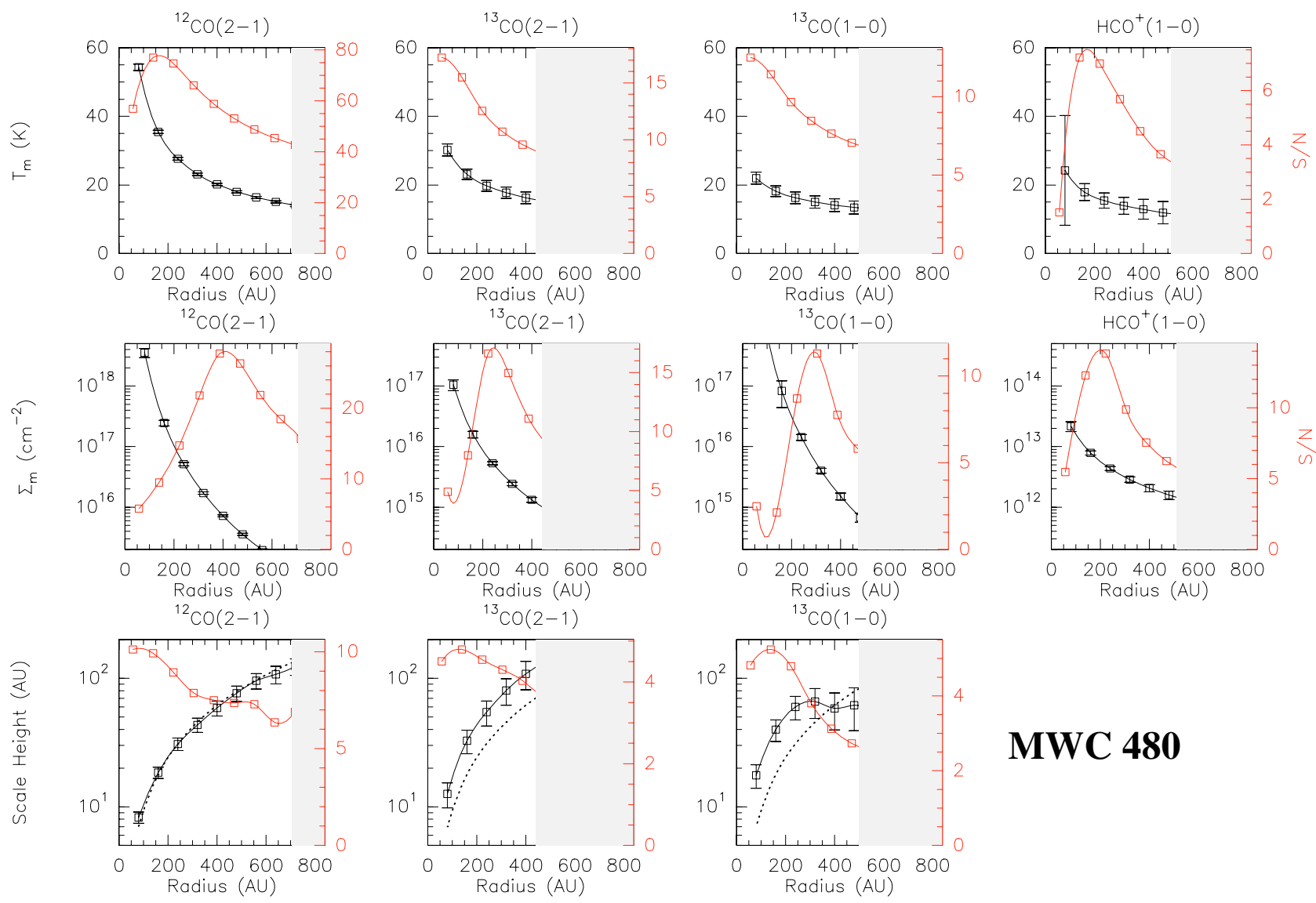

\section{MWC 480}

Fig. 6. From left to right ${ }^{12} \mathrm{CO} J=2 \rightarrow 1,{ }^{13} \mathrm{CO} J=2 \rightarrow 1,{ }^{13} \mathrm{CO} J=1 \rightarrow 0$ and $\mathrm{HCO}^{+} J=1 \rightarrow 0$ in MWC 480. Top: temperature $T_{m}$ (black curve, left axis) and signal-to-noise ratio for the temperature $T_{m} / \delta T_{m}$ (grey curve, right axis) as a function of reference radius $R_{T}$. Middle: surface density $\Sigma_{m}$ (left axis) and signal-to-noise ratio for the surface density $\Sigma_{m} / \delta \Sigma_{m}$ (right axis) as a function of reference radius $R_{\Sigma}$. Bottom: scale height $h$ (left axis) and signal-to-noise ratio for the scale height $h / \delta h$ (right axis) as a function of reference radius $R_{h}$. As for LkCa 15 , temperature and surface density are adjusted separately for $\mathrm{HCO}^{+}$.

factor), i.e., $\sim 10 \mathrm{AU}$. This super-resolution approximately matches the expected displacements due to Keplerian rotation,

$\delta r=2 r \frac{\delta v}{v} \simeq 10 \mathrm{AU}$ at $r=100 \mathrm{AU}$,

for $\delta v=0.15 \mathrm{~km} \mathrm{~s}^{-1}$. Note that in Eq. (4), the value to be used for $\delta v$ is approximately the largest of the local line width and the spectral resolution. As a result, the scale height has a measurable effect on the images, even though the flaring parameter is difficult to constrain. Actually, because of the effect of the scale height on the disk images, if a simple hydrostatic equilibrium is assumed instead of handling the scale height separately, it will result in a bias in the temperature law since one is then introducing a coupling between the vertical distribution of the molecules and the temperature, which is vertically isothermal in our model. Finally, note that because the derivation of the scale height depends on the differing inclinations of the optically thick parts, there is also a coupling between the surface density and the scale height derivation. This coupling is specially strong for disks close to edge-on.

\subsubsection{Line width}

Line broadening results from a combination of thermal and turbulent velocities

$\Delta V=\sqrt{\frac{2 k T}{m}+v_{\mathrm{turb}}^{2}}$.
However, an accurate representation of the thermal broadening requires a correct fitting of the gas temperature. This can become problematic when dealing with lines that are sub-thermally excited. Accordingly, our code offers two different parameterizations of the line width. The first one is

$\Delta V=\sqrt{\frac{2 k T}{m}+\mathrm{d} V^{2}}$, i.e., $\mathrm{d} V=v_{\text {turb }}$,

which corresponds, for $e_{v}=0\left(v_{\text {turb }}=c t e\right)$, to the parametrization used in our previous papers. The second one is

$\Delta V=\mathrm{d} V=\mathrm{d} V_{m}\left(r / R_{\mathrm{d} V}\right)^{-e_{v}}$

i.e., we do not separate the thermal component from the total line width. This offers the advantage of providing values that are not biased when the kinetic temperature is difficult to constrain (as can happen for lines that are not at LTE). We use this new description in this paper.

Note that all the line widths we quote are half-width at $1 / e$. This allows a direct comparison with the thermal velocity, $\sqrt{2 k T / m}$, but one should multiply by $2 \sqrt{\ln (2)}=1.66$ to convert to FWHM. As described before, the line width is also coupled to the scale height. 


\subsection{Minimization technique}

Using the disk parametrization described above, we have improved several aspects of the method originally developed by Dartois et al. (2003).

\subsubsection{Minimization method and error bars}

The comparison between model and data and the $\chi^{2}$ minimization is always performed inside the $U V$ plane, using natural weighting (Guilloteau \& Dutrey 1998). We have implemented a modified Levenberg-Marquardt minimization scheme to search for the minimum, and we use the Hessian to derive the error bars. This scheme is much faster than the grid search technique used previously, and less prone to local minima. It allows us to simultaneously fit more parameters, and thus provide a better estimate of the errors because the coupling between parameters is taken into account.

However, the current fit method does not handle asymmetric error bars, which happen in skewed distributions: this limitation should not be ignored when considering the errors on $\Sigma$, $T$ or $R_{\text {out }}$. The quoted error bars include thermal noise only, but do not include calibration errors (in phase and in amplitude). Amplitude calibration errors will directly affect the absolute values of the temperature or the surface density (as a scaling factor, but not the exponent), while phase errors can introduce a seeing effect. The latter effect is very small for line analysis because molecular disks are large enough, but it starts to be significant for dust disks. Our careful calibration procedure brings the amplitude effect to below $10 \%$. All other parameters are essentially unaffected by calibration errors.

\subsubsection{Continuum handling}

The speed improvement allowed us to treat the continuum emission more properly. Even though the continuum brightness is small compared to the line brightness, continuum emission cannot be ignored when trying to retrieve parameters from the line emission because it appears systematically in all spectral channels. A simultaneous fit of the continuum emission with its own physical parameters will properly take into account these biases. We performed this by adding a channel dedicated to the continuum emission to the spectral $U V$ table. The global $\chi^{2}$ is given by the sum $\chi^{2}=\chi_{\mathrm{C}}^{2}+\chi_{\mathrm{L}}^{2}$, where $\chi_{\mathrm{C}}^{2}$ and $\chi_{\mathrm{L}}^{2}$ are the $\chi^{2}$ for the continuum and for the line minimization, respectively. The continuum emission is fitted using the following parameters: $R_{\text {Cint }}$, $R_{\text {Cout }}$, the inner and outer radii of the dust distribution, $\Sigma_{\mathrm{d}}$ and $p_{\mathrm{d}}$, the surface density power law, and $T_{\mathrm{d}}$ and $q_{\mathrm{d}}$, the dust temperature power law. Note that the representation of the continuum needs only be adequate within the noise provided by the spectral (total) line width, about 10 to $20 \mathrm{MHz}$, rather than for the whole receiver bandwidth $(600 \mathrm{MHz})$. A simplified model is thus often acceptable, as shown below.

We also compared this robust method with a simplified (and faster) one in which we subtract, inside the $U V$ plane, the continuum emission from the line emission before fitting. This subtraction is not perfect: for the optically thick regions (which always occupy some fraction of the line emission) the continuum should not be subtracted. However, unless the disk is seen face on, because of the Keplerian shear, at each velocity, the lineemitting/absorbing region only occupies a small fraction of the disk area. This fraction is at most of the order of the ratio of the local line width to the projected rotation velocity at the disk edge, in practice less than $10-15 \%$ for the disks we considered.
Accordingly, the error made by subtracting the whole continuum emission in every channel is quite small.

We verified that this simplified method gives, as expected, the same results as the robust one. We used it, as it is significantly faster. This better handling of the continuum allowed us to improve on the mass determination of MWC 480 (Simon et al. 2000), where the continuum is relatively strong.

\subsubsection{Multi-line fitting}

The code has also been adapted to allow the minimization of more than one data set at a time. This has been used to simultaneously fit the ${ }^{13} \mathrm{CO} J=1 \rightarrow 0$ and $J=2 \rightarrow 1$ transitions. For multi-line fitting, the temperature is derived from the line ratio. The temperature is determined in the disk region where both lines are optically thin. In this case, the fit is much more accurate than from a single line observation, if there is no significant vertical temperature gradient. When a temperature gradient is present, the derived temperature will reflect an "average" temperature weighted by the thermal noise.

\section{Results}

\subsection{Interpretation of the power law model}

The parametric model would perfectly describe the line emission from a molecule in LTE in a vertically isothermal disk that is in hydrostatic equilibrium, with a power law for the kinetic temperature and surface density, and constant molecular abundance. In such a case, $e_{v}=q / 2$ if the local line width is thermal, and $h=q / 2-1-v$ and $h_{0}=\left(\sqrt{2 k T_{0} / \bar{m}}\right) / V_{0}$ (using the same reference radius $R_{h}=R_{T}=R_{v}, \bar{m}$ being the mean molecular weight). If such a disk were chemically homogeneous, all molecular lines would yield the same results for the 17 parameters (after correction of $\Sigma_{m}$ for the molecule abundance). Differences between the parameters derived from several transitions will reflect departures from such an ideal situation.

For example, the geometric parameters PA and $i$ should all be identical, as should be the kinematic parameters $V_{\text {disk }}, V_{0}, v$ (and we should have $v=0.50$ for Keplerian rotation). $X_{0}, Y_{0}$ should reflect the absolute astrometric accuracy of the IRAM interferometer. Different values for $T_{m}$ from several transitions for the same molecule, or its isotopologues, may reveal vertical temperature gradients, or, in the case of non-LTE excitation, density gradients, since such transitions probe different regions of the disk. The values of $T_{m}$ from different molecules may also provide constraints on the density because of the different critical densities. And, more directly, the values of $\Sigma_{m}, p_{m}, R_{\text {in }}$, and $R_{\text {out }}$ will reflect the chemical composition of the disk as a function of the radius.

\subsection{Geometric and kinematic parameters}

Figure 7 shows the geometric and kinematic parameters PA, $i$, and the rotation velocity $V_{100}$ derived from the observed transitions in DM Tau, LkCa 15, and MWC 480. The grey area represents the $1 \sigma$ range of the mean value computed from the $\mathrm{CO}$ lines. As expected, the derived values are in very good agreement altogether. In particular, the distribution of the derived values and the error bars are consistent with a statistical scattering, meaning that: i) the observed lines come from the same disk, ii) the error bars have the good magnitude, and iii) there is no bias in the determination of the parameters. This analysis of the "basic" 

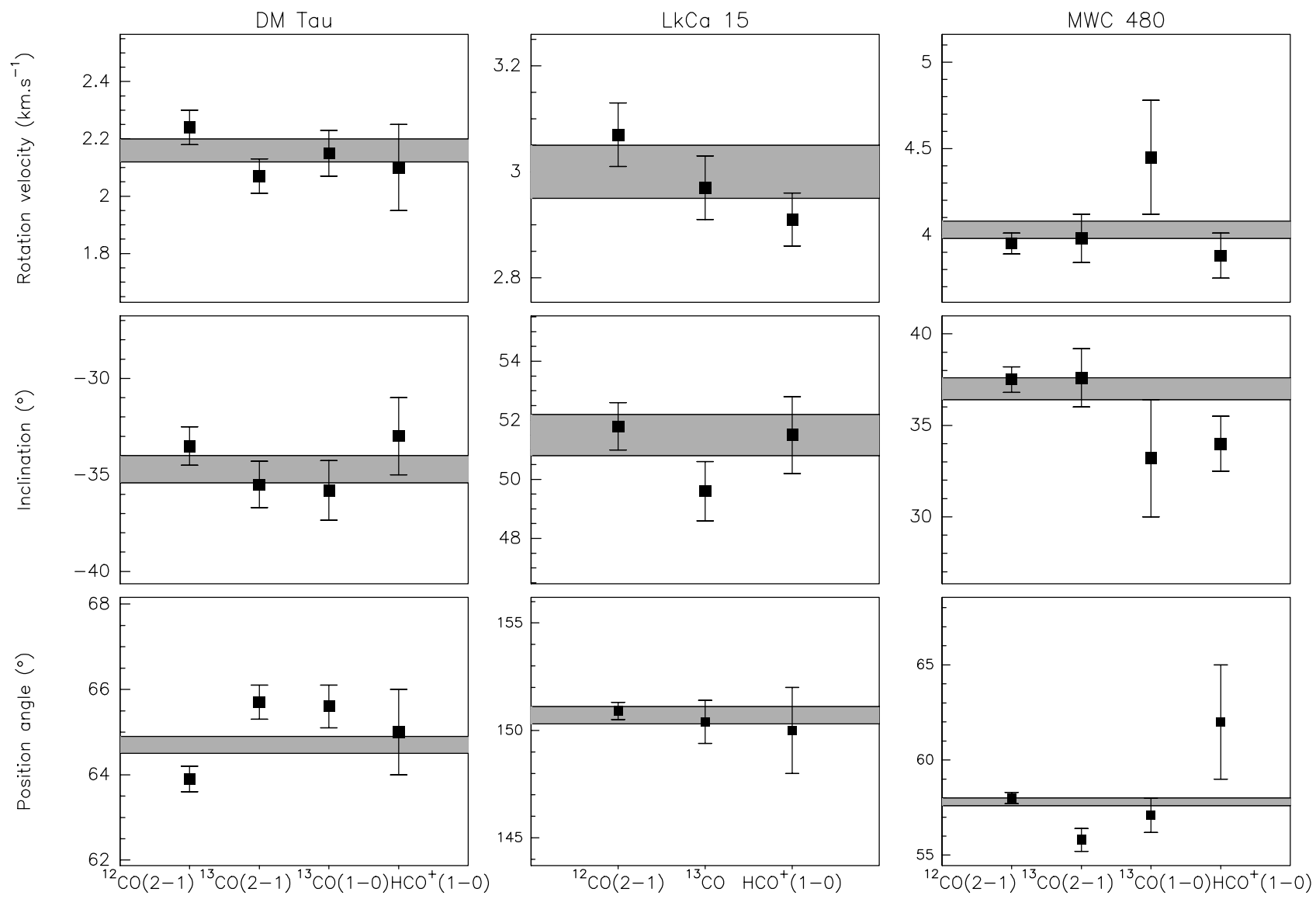

Fig. 7. From left to right: kinetic and geometric parameters derived for DM Tau, LkCa 15, and MWC 480. From top to bottom: rotation velocity at $100 \mathrm{AU}$, inclination and position angle. For DM Tau and MWC 480, the error bars represent (from left to right) the value of the parameter measured from the ${ }^{12} \mathrm{CO} J=2 \rightarrow 1,{ }^{13} \mathrm{CO} J=2 \rightarrow 1,{ }^{13} \mathrm{CO} J=1 \rightarrow 0$, and $\mathrm{HCO}^{+} J=1 \rightarrow 0$ lines. For LkCa 15 (from left to right), the values are derived from the ${ }^{12} \mathrm{CO} J=2 \rightarrow 1$ line, from the simultaneous fit of the ${ }^{13} \mathrm{CO} J=2 \rightarrow 1$ and the ${ }^{13} \mathrm{CO} J=1 \rightarrow 0$ lines, and from the $\mathrm{HCO}^{+}$ $J=1 \rightarrow 0$ line. In all three sources, the grey area represents $\pm 1 \sigma$ around the mean of the values of the CO isotopes.

parameters is of prime interest, because it shows that the analysis method is robust, and that the error bars have a real physical meaning. Although noisier, the kinematic and geometric parameters determined from $\mathrm{HCO}^{+}$are in agreement with those found from $\mathrm{CO}$. We thus used the $\mathrm{CO}$-derived values to determine the surface density and excitation temperature of $\mathrm{HCO}^{+}$.

\subsection{Results}

Tables 3-5, present the best-fit results for DM Tau, $\mathrm{LkCa} 15$, and MWC 480, respectively. Compared to previous papers (e.g., Simon et al. 2000; Duvert et al. 2000; Dutrey et al. 1998), the new fits include all the refinements of the method described in Sect. 3 and in Piétu et al. (2006). All quoted results were obtained using cylindrical coordinates and the continuum was subtracted to the spectroscopic data before analysis. All the models were obtained in the same manner. In particular, we assumed a constant line width $\left(e_{v}=0\right)$, Keplerian rotation $(v=0.5)$, and a flaring exponent $e_{h}=-1.25$ (the value for a Keplerian disk in hydrostatic equilibrium with a temperature radial dependence of 0.5$)$. This value is very close to those found by Chiang \& Goldreich (1997) for the super heated layer model. Systematic tests with different sets of free parameters ensure that the geometrical and kinematical parameters are insensitive to these assumptions. The temperature $T_{m}(r)$ is also hardly affected. The surface density $\Sigma_{m}(r)$ and outer radius $R_{\text {out }}$ depends to some extent on the assumed flaring parameter $e_{h}$. However, because of the coupling between scale height and line width, both $H_{0}$ and $\mathrm{d} V$ should be taken with some caution. The dynamical mass derived from ${ }^{12} \mathrm{CO}$ also depends weakly on the assumed scale height, since we derive the inclinations from the apparent $\mathrm{CO}$ surface.

The differences between the values reported here for DM Tau and those published by Dartois et al. (2003) come from the different assumptions in the analysis. Dartois et al. (2003) assumed hydrostatic equilibrium, and model the line width according to Eq. (6). They also assumed $\left[{ }^{12} \mathrm{CO}\right] /\left[{ }^{13} \mathrm{CO}\right]=60$.

The ${ }^{13} \mathrm{CO}$ emission in MWC 480 is strong enough to allow a separate fitting of the $J=2 \rightarrow 1$ and $J=1 \rightarrow 0$ lines. We thus present separate results for each line. We also present a result in which both lines are fitted together: as expected in this case, the derived temperature is in between the results from the $J=2 \rightarrow 1$ and $J=1 \rightarrow 0$ transitions. However, for $\mathrm{LkCa} 15$, the ${ }^{13} \mathrm{CO}$ emission is too weak for a separate analysis: only the results from the simultaneous fitting of both ${ }^{13} \mathrm{CO}$ lines is presented. For $\mathrm{HCO}^{+}$in MWC 480, the signal-to-noise ratio did not allow an independent derivation of the temperature $T_{m}$ : we used the law derived from ${ }^{13} \mathrm{CO}$. A similar problem occurs in $\mathrm{LkCa} 15$ : for simplicity, we used $T_{m}=19 \mathrm{~K}$ and $q_{m}=0.38$, values compatible with the $\mathrm{CO}$ results, although there is a solution marginally better $(1.7 \sigma)$ with slightly lower temperatures $\left(T_{m}=13 \mathrm{~K}\right)$, with a somewhat steeper surface density gradient. 
Table 3. Best parameters for DM Tau.

\begin{tabular}{|c|c|c|c|c|c|}
\hline (1) & (2) & (3) & (4) & (5) & (6) \\
\hline Lines & ${ }^{12} \mathrm{CO} J=2 \rightarrow 1$ & ${ }^{13} \mathrm{CO} J=2 \rightarrow 1$ & ${ }^{13} \mathrm{CO} J=1 \rightarrow 0$ & Mean & $\mathrm{HCO}^{+} J=1 \rightarrow 0$ \\
\hline Systemic velocity, $V_{\mathrm{LSR}}\left(\mathrm{km} \mathrm{s}^{-1}\right)$ & $6.026 \pm 0.002$ & $6.031 \pm 0.003$ & $6.088 \pm 0.004$ & $6.038 \pm 0.002$ & $6.01 \pm 0.01$ \\
\hline Orientation, $\mathrm{PA}\left({ }^{\circ}\right)$ & $63.9 \pm 0.3$ & $65.7 \pm 0.4$ & $65.6 \pm 0.5$ & $64.7 \pm 0.2$ & $65 \pm 1$ \\
\hline \multirow[t]{2}{*}{ Inclination, $i\left(^{\circ}\right)$} & $-33.5 \pm 1.0$ & $-35.5 \pm 1.2$ & $-35.8 \pm 1.5$ & $-34.7 \pm 0.7$ & $-33 \pm 2$ \\
\hline & Velocity law: & $V(r)=V_{100}\left(\frac{r}{100 \mathrm{AU}}\right)^{-l}$ & & & \\
\hline Velocity at $100 \mathrm{AU}, V_{100}\left(\mathrm{~km} \mathrm{~s}^{-1}\right)$ & $2.24 \pm 0.06$ & $2.07 \pm 0.06$ & $2.15 \pm 0.08$ & $2.16 \pm 0.04$ & $2.10 \pm 0.15$ \\
\hline Stellar mass, $M_{*}\left(M_{\odot}\right)$ & $0.56 \pm 0.03$ & $0.48 \pm 0.03$ & $0.52 \pm 0.04$ & $0.53 \pm 0.02$ & $0.5 \pm 0.1$ \\
\hline Surface density at $300 \mathrm{AU},\left(\mathrm{cm}^{-2}\right)$ & $1.4 \pm 0.6 \times 10^{17}$ & $5.3 \pm 1.1 \times 10^{15}$ & $5.9 \pm 0.6 \times 10^{15}$ & & $1.1 \pm 0.3 \times 10^{13}$ \\
\hline Exponent $p$ & $3.8 \pm 0.3$ & $3.4 \pm 0.4$ & $2.8 \pm 0.3$ & & $2.3 \pm 0.4$ \\
\hline Outer radius $R_{\text {out }},(\mathrm{AU})$ & $890 \pm 7$ & $720 \pm 19$ & $760 \pm 22$ & & $800 \pm 30$ \\
\hline Temperature at $100 \mathrm{AU},(\mathrm{K})$ & $26.0 \pm 0.5$ & $14.5 \pm 0.5$ & $8.0 \pm 0.3$ & & $14 \pm 2$ \\
\hline Exponent $q$ & $0.49 \pm 0.01$ & $0.12 \pm 0.03$ & $-0.34 \pm 0.07$ & & $0.40 \pm 0.1$ \\
\hline $\mathrm{d} V\left(\mathrm{~km} \mathrm{~s}^{-1}\right)$ & $0.12 \pm 0.01$ & $0.15 \pm 0.01$ & $0.20 \pm 0.01$ & & $0.13 \pm 0.01$ \\
\hline Scale height at $100 \mathrm{AU},(\mathrm{AU})$ & $30 \pm 1.1$ & $28 \pm 4$ & $28 \pm 5$ & & $(19 \pm 8)$ \\
\hline
\end{tabular}

Column (1) contains the parameter name. Columns (2), (3), (4) indicate the parameters derived from ${ }^{12} \mathrm{CO} J=2 \rightarrow 1,{ }^{13} \mathrm{CO} J=2 \rightarrow 1$, and ${ }^{13} \mathrm{CO} J=1 \rightarrow 0$, respectively. Only ${ }^{12} \mathrm{CO}$ constrain the sign of the inclination. Column (5) indicates the mean value of the kinematic and geometric parameters. Column (6) indicate the parameters derived from $\mathrm{HCO}^{+} J=1 \rightarrow 0$, obtained using a fixed inclination, orientation, and stellar mass. For $\mathrm{HCO}^{+}$, because of the lower resolution, the error on the temperature and surface density include contributions from the uncertainty on the exponent. $T_{m}$ is better constrained at $300 \mathrm{AU}(9.0 \pm 0.5 \mathrm{~K})$, and $\Sigma_{m}$ at $500 \mathrm{AU}\left(3.2 \pm 0.5 \times 10^{12} \mathrm{~cm}^{-2}\right)$.

Table 4. Best parameters for $\mathrm{LkCa} 15$.

\begin{tabular}{|c|c|c|c|c|}
\hline (1) & $(2)$ & (3) & (4) & $(5)$ \\
\hline Lines & ${ }^{12} \mathrm{CO} J=2 \rightarrow 1$ & ${ }^{13} \mathrm{CO}$ & Mean & $\mathrm{HCO}^{+} \quad J=1 \rightarrow 0$ \\
\hline Systemic velocity, $V_{\mathrm{LSR}}\left(\mathrm{km} \mathrm{s}^{-1}\right)$ & $6.29 \pm 0.01$ & $6.33 \pm 0.02$ & $6.30 \pm 0.01$ & $6.26 \pm 0.04$ \\
\hline Orientation, $\mathrm{PA}\left({ }^{\circ}\right)$ & $150.9 \pm 0.4$ & $150.4 \pm 1.0$ & $150.7 \pm 0.4$ & $150 \pm 2$ \\
\hline Inclination, $i\left(^{\circ}\right)$ & $51.8 \pm 0.8$ & $49.6 \pm 1.0$ & $51.5 \pm 0.7$ & $51.5 \pm 1.3$ or $46 \pm 3$ \\
\hline \multicolumn{5}{|l|}{ Velocity law: } \\
\hline Velocity at $100 \mathrm{AU}, V_{100}\left(\mathrm{~km} \mathrm{~s}^{-1}\right)$ & $3.07 \pm 0.06$ & $2.97 \pm 0.06$ & $3.00 \pm 0.05$ & \multirow[t]{2}{*}{$2.91 \pm 0.05$ or $3.11 \pm 0.15$} \\
\hline Stellar mass, $\mathrm{M}_{*}\left(M_{\odot}\right)$ & $1.06 \pm 0.04$ & $0.99 \pm 0.05$ & $1.01 \pm 0.03$ & \\
\hline Surface density at $300 \mathrm{AU},\left(\mathrm{cm}^{-2}\right)$ & $2.9 \pm 0.1 \times 10^{16}$ & $2.6 \pm 0.2 \times 10^{15}$ & & $8.0 \pm 0.5 \times 10^{12}$ \\
\hline Exponent $p$ & $4.4 \pm 0.3$ & $1.50 \pm 0.15$ & & $2.5 \pm 0.3$ \\
\hline Outer radius $R_{\text {out }},(\mathrm{AU})$ & $905 \pm 40$ & $550 \pm 20$ & & $660 \pm 60$ \\
\hline Temperature at $100 \mathrm{AU},(\mathrm{K})$ & $22.1 \pm 0.5$ & $21.2 \pm 1.7$ & & [19] \\
\hline Exponent $q$ & $0.37 \pm 0.02$ & $0.40 \pm 0.10$ & & {$[0.38]$} \\
\hline $\mathrm{d} V\left(\mathrm{~km} \mathrm{~s}^{-1}\right)$ & $0.19 \pm 0.01$ & $0.29 \pm 0.03$ & & $0.23 \pm 0.04$ \\
\hline Scale height at $100 \mathrm{AU},(\mathrm{AU})$ & $19 \pm 1$ & $17 \pm 3$ & & \\
\hline
\end{tabular}

Column (1) contains the parameter name. Columns (2), (3) indicate the parameters derived from ${ }^{12} \mathrm{CO} J=2 \rightarrow 1$, and a combined fit of the ${ }^{13} \mathrm{CO} J=2 \rightarrow 1$ and ${ }^{13} \mathrm{CO} J=1 \rightarrow 0$, respectively. Column (4) indicates the mean value of the kinematic and geometric parameters. Column (5) indicates the $\mathrm{HCO}^{+}$results: we used a fixed temperature law compatible with the $\mathrm{CO}$ isotopologue results (see text).

\section{Discussion}

We discuss here the physical implications of the results presented in the preceding section. The following subsections focus on the measurement of star masses, of the vertical temperature gradient, of the hydrostatic scale heights, of the $\mathrm{CO}$ abundances and $\mathrm{CO}$ outer radii, and of the $\mathrm{HCO}^{+}$distribution.

\subsection{CO dynamical masses}

Table 6 shows a comparison of the mass determination obtained in this work and in Simon et al. (2000). The subtraction of the continuum has slightly improved the mass determination in the case of MWC 480. Simon et al. (2000) used the old solar metallicity $(Z=0.02)$ pre-main-sequence (PMS) models. In Fig. 8, we also present results for a metallicity $Z=0.01$, much closer to the new solar metallicity (0.0126, see Asplund et al. 2004; Grevesse et al. 2005, and references therein). LkCa 15 is in agreement with models of both metallicities $\left(M=0.95 \pm 0.05 M_{\odot}, t=5-6 \mathrm{Myr}\right.$ and $1.1 \pm 0.1 M_{\odot}, t=4-6 \mathrm{Myr}$, for $Z=0.01$ and $Z=0.02$, respectively). In fact, the measured dynamical mass does not provide any strong constraint on the location of $\mathrm{LkCa} 15$ in this diagram (since the PMS tracks are almost parallel to the y-axis for stars with $0.5<M_{*} / M_{\odot}<1$ in this distance independent HR diagram), but serves as a consistency check. However for MWC 480, either the metallicity is $Z=0.01$ (i.e., nearly solar), in which case the derived dynamical mass is in agreement with the Siess et al. (2000) tracks $\left(M=1.8 M_{\odot}, t=8 \mathrm{Myr}\right)$, or the metallicity is 0.02 and MWC 480 would be located at a larger distance as suggested by Simon et al. (2000) (mass 1.9-2.0 $M_{\odot}$, 
Table 5. Best parameters for MWC 480.

\begin{tabular}{lccccc}
\hline \hline$(1)$ & $(2)$ & $(3)$ & $(4)$ & $(5)$ & $(6)$ \\
Lines & ${ }^{12} \mathrm{CO} J=2 \rightarrow 1$ & ${ }^{13} \mathrm{CO} J=2 \rightarrow 1$ & ${ }^{13} \mathrm{CO} J=1 \rightarrow 0$ & ${ }^{13} \mathrm{CO}$ & $\mathrm{HCO}^{+} J=1 \rightarrow 0$ \\
\hline Systemic velocity, $V_{\mathrm{LSR}}\left(\mathrm{km} \mathrm{s}^{-1}\right)$ & $5.076 \pm 0.003$ & $5.16 \pm 0.02$ & $5.17 \pm 0.02$ & $5.084 \pm 0.003$ & $5.11 \pm 0.04$ \\
Orientation, $\mathrm{PA}\left(^{\circ}\right)$ & $58.0 \pm 0.3$ & $55.8 \pm 0.6$ & $57.1 \pm 0.9$ & $57.8 \pm 0.2$ & $62 \pm 3$ \\
Inclination, $i\left(^{\circ}\right)$ & $37.5 \pm 0.7$ & $37.6 \pm 1.6$ & $33.2 \pm 3.2$ & $37.0 \pm 0.6$ & $34.0 \pm 1.5$ \\
\hline
\end{tabular}

Velocity law: $\quad V(r)=V_{100}\left(\frac{r}{100 \mathrm{AU}}\right)^{-v}$

\begin{tabular}{lccccc} 
Velocity at $100 \mathrm{AU}, V_{100}\left(\mathrm{~km} \mathrm{~s}^{-1}\right)$ & $3.95 \pm 0.06$ & $3.98 \pm 0.14$ & $4.45 \pm 0.33$ & $4.03 \pm 0.05$ & $3.88 \pm 0.13$ \\
Stellar mass, $\mathrm{M}_{*}\left(M_{\odot}\right)$ & $1.76 \pm 0.06$ & $1.65 \pm 0.15$ & $2.47 \pm 0.23$ & $1.83 \pm 0.05$ & $1.70 \pm 0.12$ \\
\hline Surface density at $300 \mathrm{AU},\left(\mathrm{cm}^{-2}\right)$ & $3.6 \pm 0.2 \times 10^{16}$ & $3.3 \pm 0.4 \times 10^{15}$ & $5.3 \pm 0.9 \times 10^{15}$ & $4.5 \pm 0.3 \times 10^{15}$ & $3.4 \pm 0.3 \times 10^{12}$ \\
Exponent $p$ & $4.7 \pm 0.3$ & $3.0 \pm 0.4$ & $4.5 \pm 0.9$ & $3.9 \pm 0.3$ & $1.5 \pm 0.2$ \\
Outer radius $R_{\text {out }},(\mathrm{AU})$ & $740 \pm 15$ & $450 \pm 15$ & $520 \pm 70$ & $480 \pm 20$ & $520 \pm 50$ \\
Temperature at $100 \mathrm{AU},(\mathrm{K})$ & $48 \pm 1$ & $28 \pm 2$ & $21 \pm 4$ & $23 \pm 1$ & $(15 \pm 6)$ \\
Exponent $q$ & $0.65 \pm 0.02$ & $0.37 \pm 0.08$ & $0.28 \pm 0.09$ & $0.37 \pm 0.04$ & $(0.6 \pm 0.4)$ \\
$\mathrm{d} V\left(\mathrm{~km} \mathrm{~s}^{-1}\right.$ ) & $0.25 \pm 0.01$ & $0.25 \pm 0.02$ & $0.15 \pm 0.03$ & $0.21 \pm 0.02$ & $0.33 \pm 0.07$ \\
Scale height at $100 \mathrm{AU},(\mathrm{AU})$ & $10 \pm 1.1$ & $18 \pm 4$ & $20 \pm 2$ & $19 \pm 2$ & \\
\hline
\end{tabular}

Column (1) contains the parameter name. Columns (2), (3), (4) indicate the parameters derived from ${ }^{12} \mathrm{CO} J=2 \rightarrow 1$, ${ }^{13} \mathrm{CO} J=2 \rightarrow 1$, and ${ }^{13} \mathrm{CO} J=1 \rightarrow 0$, respectively. Column (5) indicates the mean value of the kinematic and geometric parameters $\left(V_{\mathrm{LSR}}, \mathrm{PA}, i, V_{100}\right)$. For the other parameters, Column (5) indicates the results of a simultaneous fit of the two ${ }^{13} \mathrm{CO}$ transitions as for LkCa 15 . Column (6) indicates the $\mathrm{HCO}^{+}$ results: as the temperature is poorly constrained, the surface densities were derived assuming the temperature given by ${ }^{13} \mathrm{CO} J=1 \rightarrow 0$. The surface density is best determined at $250 \mathrm{AU}: 4.3 \pm 0.3 \times 10^{12} \mathrm{~cm}^{-2}$.

age $\sim 7 \mathrm{Myr}$, and the distance would need to be increased by about $10 \%$ ).

\subsection{Vertical temperature gradient}

The primary goal of these observations was to confirm the findings of Dartois et al. (2003): the existence of a vertical temperature gradient. Figure 9 shows the temperatures deduced from the various $\mathrm{CO}$ lines versus the star effective temperatures in all sources of the sample. We confirm the DM Tau results with this slightly different analysis. MWC 480 also presents a significant temperature difference between ${ }^{12} \mathrm{CO}$ and ${ }^{13} \mathrm{CO}$. However, in $\mathrm{LkCa} 15$, there is no clear evidence of a temperature gradient. As a general trend, the hotter stars of the sample, namely the Herbig Ae stars, exhibit larger kinetic temperature in the disks, not only at their surfaces, but also for their interiors (see also Sect. 5.8 for AB Aur). For MWC 480, even if the surface appears hotter, a significant fraction of the disk (for $r>200 \mathrm{AU}$ ) remains at temperature below the $\mathrm{CO}$ freeze-out temperature.

The comparison between the three sources can be understood by noting that the surface densities of ${ }^{13} \mathrm{CO}$ decreases from DM Tau to MWC 480 and LkCa 15 (see Sect. 5.8). Moreover, the temperature is lower in DM Tau, resulting in higher opacities for the $J=2 \rightarrow 1$ and $J=1 \rightarrow 0$ transitions than in the other sources. As a result, the location of the $\tau=1$ surface in the ${ }^{13} \mathrm{CO}$ lines differ in the three sources. In DM Tau, this surface is above (for the $J=2 \rightarrow 1$ line) or at (for the $J=1 \rightarrow 0$ line) the disk plane (as seen from the observer). A similar behavior is observed in MWC 480. In LkCa 15, the ${ }^{13} \mathrm{CO}$ lines are nearly optically thin throughout the disk, and the temperature is accurately determined by the $J=2 \rightarrow 1 / J=1 \rightarrow 0$ line ratio. The lack of difference between the ${ }^{12} \mathrm{CO}$ and ${ }^{13} \mathrm{CO}$ results indicate both lines are formed in a similar region: there must be little ${ }^{13} \mathrm{CO}$ at temperatures much lower than indicated by ${ }^{12} \mathrm{CO}$.

\subsection{CO surface density and outer radius}

There are significant differences in surface densities among the three sources. At $300 \mathrm{AU}$, the measured surface densities of ${ }^{13} \mathrm{CO}$ range from $\sim 2.5 \times 10^{15} \mathrm{~cm}^{-2}$ in LkCa 15 to $\sim 3.2 \times$ $10^{15} \mathrm{~cm}^{-2}$ in MWC 480 and $\sim 7 \times 10^{15} \mathrm{~cm}^{-2}$ in DM Tau. The variations are even larger for ${ }^{12} \mathrm{CO}$ with values $\sim 3 \times 10^{16} \mathrm{~cm}^{-2}$ in LkCa 15 and MWC 480, and 4 times larger, $14 \times 10^{16} \mathrm{~cm}^{-2}$ for DM Tau (although the latter is uncertain by a factor 2 because of the steep density gradient).

The ${ }^{12} \mathrm{CO} /{ }^{13} \mathrm{CO}$ ratio is $20 \pm 3$ in $\mathrm{DM}$ Tau near $450 \mathrm{AU}$, the distance where this ratio is best determined. It is $11 \pm 2$ at $300 \mathrm{AU}$ for $\mathrm{LkCa} 15$, and $8 \pm 2$ for MWC 480. These values are much lower than the standard ${ }^{12} \mathrm{C} /{ }^{13} \mathrm{C}$ ratio in the solar neighborhood. These low values are most likely the result of significant fractionation, as in the Taurus molecular clouds, since the typical temperatures near $400 \mathrm{AU}$ are around $12 \mathrm{~K}$ (see also Sect. 5.8).

The exponent of the surface density distribution varies significantly. For DM Tau and MWC 480, an exponent of $\sim 3.5$ is appropriate for all isotopologues. For $\mathrm{LkCa} 15$, there is a large difference between the exponent for ${ }^{13} \mathrm{CO}, 1.5$, and that for ${ }^{12} \mathrm{CO}, 4.5$. Each line, however, samples a very different region of the disk. The ${ }^{13} \mathrm{CO}$ data are most sensitive to the 200-300 AU region, while the ${ }^{12} \mathrm{CO}$ data is influenced by the outermost parts, 500-700 AU (because of the opacity of the ${ }^{12} \mathrm{CO}$ line) and up to the outer disk radius. The difference in exponent may thus indicate a steepening of the ${ }^{12} \mathrm{CO}$ surface density distribution at radii larger than $>500 \mathrm{AU}$. At such radii, the ${ }^{12} \mathrm{CO}$ emission becomes optically thin, and better constrains the surface density than ${ }^{13} \mathrm{CO}$. For DM Tau and MWC 480, the surface density of CO falls faster with radius than usually assumed for molecular disks $\left(p \sim 1-1.5\right.$, for $\left.\mathrm{H}_{2}\right)$. Note that the uncertainty on temperature law only weakly affects the surface density derivation, especially for the $J=2 \rightarrow 1$ transition for which the emission is essentially proportional to $\Sigma_{m}$ for the considered temperature range in the optically thin regime. This apparent steepening could reflect a change in the $\left(\mathrm{H}_{2}\right)$ surface density distribution in the disk. However, it can also be a result of chemical effects. 

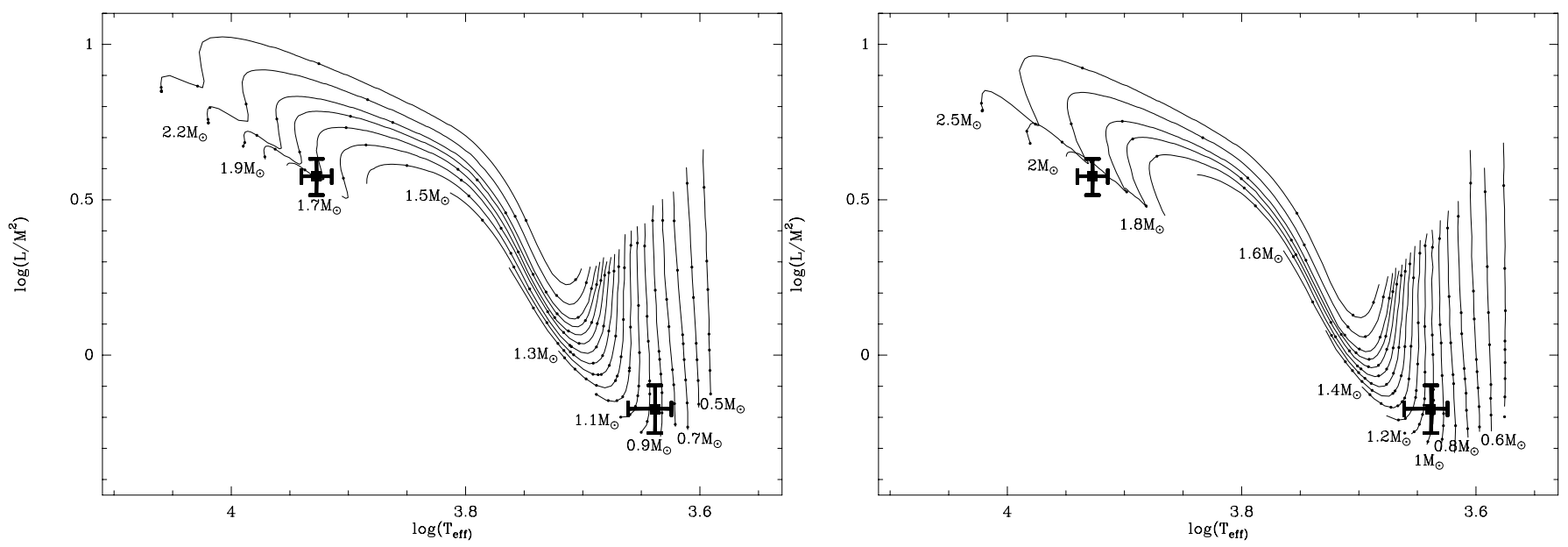

Fig. 8. Summary of the dynamical mass measurement for the sources of this sample. As in Simon et al. (2000), the results are shown in a distancecorrected HR diagram $\left(L / M^{2}\right.$ versus $\left.T_{\text {eff }}\right)$. New results have been obtained for MWC 480 and LkCa 15 . They are all compatible with the previous ones. In the case of MWC 480, the improvement in the mass determination is due to a better fit of the continuum, as explained in Sect. 3.3.2. Left: tracks for a stellar metallicity $Z=0.01$. Right: tracks for a stellar metallicity $Z=0.02$. The models have been computed by Siess et al. (2000). Dots starts at $1 \mathrm{Myr}$ and are spaced by $1 \mathrm{Myr}$. The tracks range from 0.5 to 2.0 by $0.1 M_{\odot}$, with the 2.2 and $2.5 M_{\odot}$ tracks added. For $T_{\text {eff }}$, the error bars are assumed to be $+/$ - one sub spectral type class.

The isotropic ambient UV field will tend to photo-dissociate CO isotopologues near the disk outer edge. Another effect is that, because of the lower temperatures, depletion onto dust grains may be more effective at larger radii (although the lower densities result in a larger timescale for sticking onto grains).

In all three sources, the outer radius in ${ }^{13} \mathrm{CO}$ is significantly smaller than in ${ }^{12} \mathrm{CO}$. In $\mathrm{LkCa} 15$, the large difference may be partly attributed to the change in the exponent of the density law. However, if we assume $p=1.5$ for ${ }^{12} \mathrm{CO}$, we get an outer radius of $710 \pm 10$, still significantly larger than that for ${ }^{13} \mathrm{CO}$. The effect is thus genuine.

\subsection{The LkCa 15 inner hole}

In the case of $\mathrm{LkCa} 15$, it is also important to mention its peculiar geometry: Piétu et al. (2006) have discovered a 50 AU radius cavity in the continuum emission from $\mathrm{LkCa} 15$. We checked whether there is any hint of this cavity in the CO data. Adding the inner radius as an additional free parameter yields $R_{\text {int }}=$ $13 \pm 5 \mathrm{AU}$ for ${ }^{12} \mathrm{CO}$ and $R_{\text {int }}=23 \pm 8 \mathrm{AU}$ for ${ }^{13} \mathrm{CO}$. These values are consistent with a smaller hole, or no hole at all, in $\mathrm{CO}$ and ${ }^{13} \mathrm{CO}$. The $50 \mathrm{AU}$ inner radius determined from the continuum is excluded at the $7 \sigma$ level in ${ }^{12} \mathrm{CO}$, and at the $3 \sigma$ level in ${ }^{13} \mathrm{CO}$. Moreover the fit of a hole does not significantly change the value of $p$, for both ${ }^{12} \mathrm{CO}$ and ${ }^{13} \mathrm{CO}$ emissions.

These small values indicate that the $\mathrm{CO}$ gas extends well into the continuum cavity. The cavity is thus not completely void, but still has a sufficient surface density for the ${ }^{13} \mathrm{CO}$ lines to be detected. The $\mathrm{LkCa} 15$ situation is similar to that of $\mathrm{AB}$ Aur, for which the apparent inner radius increases from ${ }^{12} \mathrm{CO}$ to ${ }^{13} \mathrm{CO}$ and to the dust emission (Piétu et al. 2005).

\section{5. $\mathrm{HCO}^{+}$surface density}

The $\mathrm{HCO}^{+}$data analysis reveals 4 major facts

- the outer radius in $\mathrm{HCO}^{+}$is similar to that in ${ }^{12} \mathrm{CO}$, and in particular it is larger than that in ${ }^{13} \mathrm{CO}$.
Table 6. Stellar masses.

\begin{tabular}{lcc}
\hline \hline Source & $\begin{array}{c}\text { Previous mass } \\
\left(M_{\odot}\right)\end{array}$ & $\begin{array}{c}\text { This work } \\
\left(M_{\odot}\right)\end{array}$ \\
\hline DM Tau & $0.55 \pm 0.03$ & $0.53 \pm 0.02$ \\
LkCa 15 & $0.97 \pm 0.03$ & $1.01 \pm 0.03$ \\
MWC 480 & $1.65 \pm 0.07$ & $1.83 \pm 0.05$ \\
\hline
\end{tabular}

See Simon et al. (2000) for the previous measurements. The old masses are based only on ${ }^{12} \mathrm{CO}$, while the new measurements are the average of all available $\mathrm{CO}$ lines.

- in the two cases where it could be determined, the excitation temperature of the $\mathrm{HCO}^{+} J=1 \rightarrow 0$ is lower than that of the ${ }^{13} \mathrm{CO} J=2 \rightarrow 1$ line.

- at $300 \mathrm{AU}$, the $\left[{ }^{13} \mathrm{CO}\right] /\left[\mathrm{HCO}^{+}\right]$ratio is $500 \pm 150(\mathrm{DM} \mathrm{Tau})$, $320 \pm 50(\mathrm{LkCa} 15)$, and $1300 \pm 200$ for MWC 480, with an additional uncertainty around $50 \%$ for these last two sources due to the adopted temperature distribution. These values are similar to, although slightly smaller than the one found by Guilloteau et al. (1999) for the circumbinary disk of GG Tau $(\sim 1500)$. On the other end, Pety et al. (2006) find a ratio of about $10^{4}$ for the low-mass disk around $\mathrm{HH} 30$, but at a smaller radius (100 AU).

- This ratio varies with radius: there is a tendency to have a somewhat flatter distribution of $\mathrm{HCO}^{+}$than of $\mathrm{CO}$ (with the exception of ${ }^{13} \mathrm{CO}$ in $\left.\mathrm{LkCa} 15\right)$.

In DM Tau, the excitation temperature falls down to about $6 \mathrm{~K}$ beyond $600 \mathrm{AU}$, while the $\mathrm{CO}$ data indicates kinetic temperature of about $10 \mathrm{~K}$ in this region. Our calibration technique ensures that this is not an artifact. It may be a hint of very low temperatures in the disk mid-plane. On the other end, it may be an indication of sub-thermal excitation. However, as the critical density for the $\mathrm{HCO}^{+} J=1 \rightarrow 0$ is only a few $10^{4} \mathrm{~cm}^{-3}$, such a sub-thermal excitation would require that the $\mathrm{HCO}^{+}$gas is located well above the disk plane. Spatially resolved observations of the $\mathrm{HCO}^{+} J=3 \rightarrow 2$ transition are required to check this possibility. 
Table 7. $\mathrm{HCO}^{+}$and $\mathrm{CO}$ outer radius.

\begin{tabular}{lccc}
\hline \hline Source & $\begin{array}{c}R_{\text {out }}\left({ }^{12} \mathrm{CO}\right) \\
(\mathrm{AU})\end{array}$ & $\begin{array}{c}R_{\text {out }}{ }^{\left({ }^{13} \mathrm{CO}\right)} \\
(\mathrm{AU})\end{array}$ & $\begin{array}{c}\mathrm{HCO}^{+} \\
(\mathrm{AU})\end{array}$ \\
\hline DM Tau & $890 \pm 7$ & $740 \pm 15$ & $800 \pm 80$ \\
LkCa 15 & $905 \pm 40$ & $550 \pm 20$ & $660 \pm 60$ \\
MWC 480 & $740 \pm 15$ & $480 \pm 20$ & $520 \pm 50$ \\
\hline
\end{tabular}

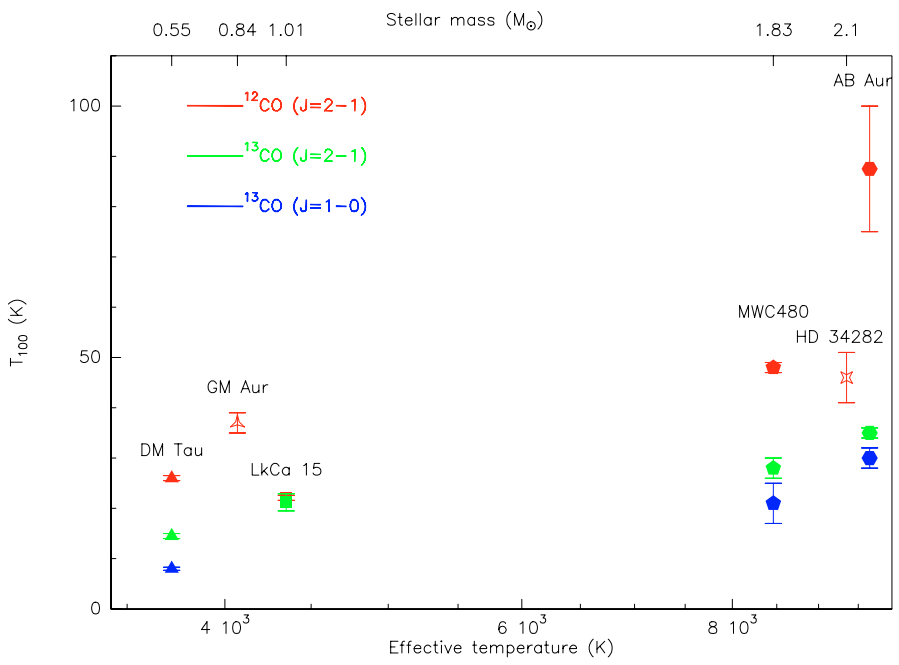

Fig. 9. Temperatures derived from the $\mathrm{CO}$ isotopes versus effective temperature of the central star. From left to right, the sources are DM Tau (filled triangles), GM Aur (empty stars), LkCa 15 (filled squares), MWC 480 (filled pentagons), HD 34282 (empty stars) and AB Aur (filled hexagons). From top to bottom, temperatures derived from ${ }^{12} \mathrm{CO} J=2 \rightarrow 1,{ }^{13} \mathrm{CO} J=2 \rightarrow 1$, and ${ }^{13} \mathrm{CO} J=1 \rightarrow 0$, respectively.

\subsection{Turbulence in outer disks}

We derive intrinsic (local) line widths ranging between 0.12 and $0.29 \mathrm{~km} \mathrm{~s}^{-1}$. When taking into account the thermal component (0.08 to $\left.0.15 \mathrm{~km} \mathrm{~s}^{-1}\right)$, from Eqs. (6) and (7) we derive turbulent widths below $0.15 \mathrm{~km} \mathrm{~s}^{-1}$. These values should be used as upper limits, since the spectral resolution used for the analysis $\left(0.2 \mathrm{~km} \mathrm{~s}^{-1}\right)$ is comparable to the derived line widths. They are nevertheless significantly smaller than the sound speed, $C_{\mathrm{s}}=0.3$ to $0.5 \mathrm{~km} \mathrm{~s}^{-1}$ in the relevant temperature and radius range. The turbulence is thus largely subsonic. A more precise analysis, using the full spectral resolution and accurate knowledge of the kinetic temperature distribution, is required for a better determination.

\subsection{Hydrostatic scale height}

We follow the approach that is described in Sect. 3.2.5 where the scale-height is not formally derived from the hydrostatic equilibrium. In a first step, the flaring exponent $e_{h}$ is taken to be equal to $e_{h}=-1.25$. Such a value is in agreement with predictions from models such as Chiang \& Goldreich (1997) and D'Alessio et al. (1999). The observed transitions indicate apparent scale heights at $100 \mathrm{AU}$ of $30 \mathrm{AU}$ in DM Tau, and $19 \mathrm{AU}$ in LkCa 15 and MWC 480 (see Tables 3-5). The only discrepant result is the value derived from ${ }^{12} \mathrm{CO}$ in MWC 480, $10 \mathrm{AU}$. In a second step, treating $e_{h}$ as a free parameter provides similar results, except for $\mathrm{LkCa} 15$, where the ${ }^{13} \mathrm{CO}$ data is best fitted by a flat $\left(e_{h}=0\right)$, but thick disk (see Fig. 5).
On the other end, using the temperature derived from the ${ }^{13} \mathrm{CO} J=2 \rightarrow 1$ transition as appropriate, we can derive the hydrostatic scale heights. These scale heights are 15, 13, and 9.5 AU, respectively for DM Tau, LkCa 15, and MWC 480, with a typical exponent $e_{h}=-1.35$, approximately $1.5-2$ times smaller than the apparent values. Although, as mentioned in Sect. 3.2.5, the scale height has to be interpreted with some caution (especially when determined from ${ }^{12} \mathrm{CO}$ ), these results suggest that $\mathrm{CO}$ has a broader vertical distribution than what is expected from an hydrostatic distribution of the gas. The current data do not allow us to identify the causes of this effect. Vertical spreading due to turbulence can be rejected as the turbulence is largely subsonic (see Sect. 5.6). The larger apparent height may be due to $\mathrm{CO}$ being more abundant in the photo-dissociation layer above the disk plane.

\subsection{Comparison with other disks and models}

Only one other source has been studied at the same level in the CO isotopologues: AB Aur by Piétu et al. (2005). The temperature behavior of the $\mathrm{AB}$ Aur disk is similar to that of the MWC 480 disk, although the AB Aur disk is warmer. Temperature derived from $\mathrm{CO}$ for all sources are summarized in Fig. 9. As expected, the disk temperatures (midplane and surface) clearly increase with the stellar luminosity. The A0/A1 star HD 34282, mapped by Piétu et al. (2003) in ${ }^{12} \mathrm{CO}$ also exhibits a hotter disk surface while the TTauri star GM Aur (Dutrey et al. 1998) has a colder disk. From Table 1 of Piétu et al. (2005), the ${ }^{13} \mathrm{CO}$ surface density in AB Aur at $300 \mathrm{AU}$ is about $2 \times 10^{16} \mathrm{~cm}^{-2}, 4$ to 8 times larger than in the DM Tau, MWC 480, or LkCa 15 disks. This larger surface density probably results from a lack of depletion onto dust grains, since the temperature in the AB Aur disk remains well above the $\mathrm{CO}$ condensation temperature at least up to $700 \mathrm{AU}$ in radius, contrary to those of DM Tau, LkCa 15, and MWC 480, which are much colder beyond 200-300 AU.

LkCa 15 differs slightly from the general picture: no clear vertical temperature gradient is observed, and the ${ }^{13} \mathrm{CO}$ surface density slope is much less steep (exponent $p=1.5$ ) and the CO isotopologues indicate a discrepant scale height behavior. This may be linked to a peculiar disk geometry: Piétu et al. (2006) have reported a $50 \mathrm{AU}$ radius cavity in the continuum emission from $\mathrm{LkCa} 15$, perhaps due to planetary formation or a low-mass companion. This peculiar geometry may affect the temperature structure of the surrounding disk, for example by shielding it from stellar radiation behind a warm, thick, inner rim. Any definite conclusion about the peculiarities of LkCa 15 seems premature at this point.

Molecular abundances in proto-planetary disks have been studied by several authors. Disks are believed to be dominated by a warm molecular layer, exposed to the UV radiation emanating from the central star. van Zadelhoff et al. (2003) showed that 2-D radiative transfer effects result in a stronger penetration of the UV field into the disk than simple 1+1D models assumed. A more refined model was published by Aikawa \& Nomura (2006), which uses time dependent chemistry taking into account the thermal structure of the disk, 2-D radiative transfer, and sticking onto grains. Their model parameters are more appropriate for DM Tau. Despite its comprehensiveness, this model fails to represent the observed surface densities: it predicts relatively shallow ( $p \simeq 1$ ) radial distribution of the surface density, in sharp contrast with the steep slope observed. However, their model only extends out to $300 \mathrm{AU}$, while the constraint we obtain on the surface densities in DM Tau mostly refers to larger radii. 
Semenov et al. (2006) point out the importance of vertical mixing in explaining the molecular surface densities. A similar study was conducted by Willacy et al. (2006), who conclude that a diffusion coefficient of order $10^{18} \mathrm{~cm}^{2} \mathrm{~s}^{-1}$ is required to bring the $\mathrm{CO}$ surface density in reasonable agreement with observations. Vertical mixing may be essential in explaining why $\mathrm{CO}$ is detected at temperature well below the freeze-out value, and also why, despite very different temperature profiles, DM Tau and MWC 480 display similar CO surface densities.

None of these models studied the ${ }^{13} \mathrm{CO}$ isotopologue. Hily-Blant et al. (2007) used a simpler PDR model to study the effects of grain size and stellar UV flux on the CO isotopologue abundances. In this static model, the self and mutual shielding of $\mathrm{H}_{2}, \mathrm{CO}$, and ${ }^{13} \mathrm{CO}$ is treated explicitly, but $\mathrm{CO}$ sticking onto grains is not included. This model shows the importance of fractionation and reproduces the observed surface densities better. In particular, from their Fig. 6 , $\left[{ }^{12} \mathrm{CO}\right] /\left[{ }^{13} \mathrm{CO}\right]$ ratio of order $10-20$ is obtained for large grains $\left(a_{+}=3 \times 10^{-4} \mathrm{~m}\right)$ and $\chi=1000(\chi$ being the enhancement over the interstellar UV field at $100 \mathrm{AU}$, with a $1 / r^{2}$ dependence), i.e., when the UV field penetration is important.

So far, no published model reproduces smaller outer radii for ${ }^{13} \mathrm{CO}$ than for ${ }^{12} \mathrm{CO}$. To do so, it seems essential to account for selective photodissociation of $\mathrm{CO}$ isotopologues by the interstellar UV radiation field, and in particular by the $U V$ radiation impinging on the disk outer edge. The disappearance of ${ }^{13} \mathrm{CO}$ at distances $\sim 500 \mathrm{AU}$ from MWC 480 and $\mathrm{LkCa} 15$ indicates that the typical extinction through the disk at this radius corresponds roughly to $A_{\mathrm{V}} \simeq 1$, although the disks extend to at least 700-900 AU. A consistency check can be done on this hypothesis: assuming a standard $A_{\mathrm{V}} / \mathrm{H}_{2}$ ratio, the ${ }^{13} \mathrm{CO}$ outer radius can be used to constrain the $\mathrm{CO}$ abundance at this distance. From Figs. 4-6, the typical ${ }^{12} \mathrm{CO}$ surface density at the ${ }^{13} \mathrm{CO}$ outer radius is $2-4 \times 10^{15} \mathrm{~cm}^{-2}$, which converts to $\mathrm{X}\left[{ }^{12} \mathrm{CO}\right] \simeq 3-6 \times 10^{-5}$, in good agreement with the expectation in this transition region. In DM Tau, $A_{\mathrm{V}} \simeq 1$ would occur near $800 \mathrm{AU}$, i.e., the (outer) DM Tau disk is more opaque than those of MWC 480 and LkCa 15. This larger opacity can result either from a larger $\mathrm{H}_{2}$ density, or from a larger $A_{\mathrm{V}} / \mathrm{H}_{2}$, i.e., more small grains in DM Tau.

All the chemical models are hampered by the need to specify the appropriate $\mathrm{H}_{2}$ density distribution and the dust properties. These parameters remain largely unknown. The values of $p$ and $R_{\text {out }}$ for the dust in LkCa 15 and MWC 480 derived by Piétu et al. (2006) differ systematically from those found here for $\mathrm{CO}$. In addition, the absolute value of the absorption coefficient of dust is also uncertain, which makes the derivation of an $\mathrm{H}_{2}$ surface density through continuum observations quite difficult. Using the parameters given by Piétu et al. (2006), we find at the dust core outer radius, $\approx 180 \mathrm{AU}$, that the $\mathrm{CO}$ abundance is $\sim 10^{-6}$ for LkCa 15 , while it is $\sim 3 \times 10^{-7}-3 \times 10^{-6}$ for MWC 480, depending on which temperature profile is adopted for the dust. These very low values, as well as the very different values of the exponent $p$ for dust and $\mathrm{CO}$, may thus indicate that the $\mathrm{H}_{2}$ surface density cannot be extrapolated by a simple power law, but instead displays a very significant change near $\sim 150$ AU. This can have a significant impact on the chemical model predictions for any specific disk.

\section{Summary}

We report new ${ }^{13} \mathrm{CO}$ observations in LkCa 15 and MWC 480 obtained with the IRAM array, and $\mathrm{HCO}^{+}$observations of $\mathrm{LkCa} 15$, MWC 480, and DM Tau. These data were analyzed together with existing ${ }^{12} \mathrm{CO}$ data. As we improved our modelling method, we also carried out the analysis on the DM Tau data again as a consistency check. By comparing these results with previous ones obtained on $\mathrm{AB}$ Aur (and to the temperatures derived from ${ }^{12} \mathrm{CO}$ observations in HD 34282 and GM Aur), this allowed us to derive the following conclusions:

- We systematically detect radial gas temperature gradients. Vertical temperature gradients are also ubiquitous in AB Aur, DM Tau, and MWC 480, but LkCa 15 is an apparent exception (perhaps due to its peculiar geometry). Disks surrounding hotter stars, such as Herbig Ae objects (MWC 480 and AB Aur), present a hotter CO surface. This behavior agrees with the prediction from models of passive irradiated disks.

- CO disks around hotter stars are also hotter around the midplane (AB Aur and to a lesser extent MWC 480). Although $\left[\mathrm{CO} / \mathrm{H}_{2}\right]$ ratios are not directly measurable, the much higher surface density of ${ }^{13} \mathrm{CO}$ in the warmest disk, $\mathrm{AB}$ Aur, indicates that depletion onto grains is an important mechanism for the colder disks. However, the CO surface density does not appear to be a monotonous function of the stellar type.

- For the more sensitive transitions, we are able to measure an apparent scale height that appears to be larger than the hydrostatic scale height. Although marginal, this result is in agreement with the expectation that molecules preferentially form in a warm photo-dissociation layer above the disk plane.

- However, in the outer part of TTauri disks ( $R \geq 300 \mathrm{AU})$ and even around MWC 480, the averaged kinetic temperature measured along the line of sight is well below the freezeout temperature of $\mathrm{CO}$, but a significant amount of $\mathrm{CO}$ is still observed. This is in contradiction with most predictions of chemical models, but would be in favor of models with vertical mixing.

- The CO surface density falls off much more rapidly with the radius than predicted by most chemical models. In the cases of DM Tau and $\mathrm{LkCa} 15$, we also observe that the ${ }^{12} \mathrm{CO}$ has a steeper surface density exponent than ${ }^{13} \mathrm{CO}$. This suggests a steepening of the surface density slope beyond 300-500 AU, but photo-dissociation, and also perhaps depletion on grains are likely to play a role.

- A change in surface density behavior is also suggested by the very different parameters (exponent $p$ and outer radius) derived from the continuum emission.

- For all disks where ${ }^{12} \mathrm{CO}$ and ${ }^{13} \mathrm{CO}$ data are available, photodissociation by the ambient UV field impinging on the disk edge is likely the appropriate mechanism to explain the smaller outer radii determined in ${ }^{13} \mathrm{CO}$ compared to that of ${ }^{12} \mathrm{CO}$.

- Except for the warmest disks, CO fractionation is an important mechanism. For DM Tau and LkCa 15, we estimate an isotopologue ratio, of order or less than 10-20 between $300 \mathrm{AU}$ and the ${ }^{13} \mathrm{CO}$ outer radius.

- At $300 \mathrm{AU}$, the $\left[{ }^{13} \mathrm{CO}\right] /\left[\mathrm{HCO}^{+}\right]$ratio is around 600 , within a factor 2 . It apparently decreases with radius.

Acknowledgements. We acknowledge all the Plateau de Bure IRAM staff for their help during the observations. We also would like to thank Guilen Oyarçabal and Rowan Smith for their participation in the fits.

\section{References}

Aikawa, Y., \& Nomura, H. 2006, ApJ, 642, 1152

Asplund, M., Grevesse, N., Sauval, A. J., Allende Prieto, C., \& Kiselman, D. 2004, A\&A, 417, 751

Chiang, E. I., \& Goldreich, P. 1997, ApJ, 490, 368 
D’Alessio, P., Calvet, N., Hartmann, L., Lizano, S., \& Cantó, J. 1999, ApJ, 527, 893

Dartois, E., Dutrey, A., \& Guilloteau, S. 2003, A\&A, 399, 773

Dullemond, C. P., \& Dominik, C. 2004, A\&A, 417, 159

Dullemond, C. P., Dominik, C., \& Natta, A. 2001, ApJ, 560, 957

Dutrey, A., Guilloteau, S., \& Simon, M. 1994, A\&A, 286, 149

Dutrey, A., Guilloteau, S., \& Guelin, M. 1997, A\&A, 317, L55

Dutrey, A., Guilloteau, S., Prato, L., et al. 1998, A\&A, 338, L63

Duvert, G., Guilloteau, S., Ménard, F., Simon, M., \& Dutrey, A. 2000, A\&A, 355,165

Grady, C. A., Woodgate, B., Bruhweiler, F. C., et al. 1999, ApJ, 523, L151

Grevesse, N., Asplund, M., \& Sauval, A. J. 2005, in EAS Publications Series, ed. G. Alecian, O. Richard, \& S. Vauclair, 21

Guilloteau, S., \& Dutrey, A. 1994, A\&A, 291, L23

Guilloteau, S., \& Dutrey, A. 1998, A\&A, 339, 467

Guilloteau, S., Dutrey, A., \& Simon, M. 1999, A\&A, 348, 570

Hartmann, L., Calvet, N., Gullbring, E., \& D’Alessio, P. 1998, ApJ, 495, 385

Hily-Blant, P., Dartois, E., Roueff, E., et al. 2007, A\&A, submitted

Kenyon, S. J., Dobrzycka, D., \& Hartmann, L. 1994, AJ, 108, 1872

Koerner, D. W., Sargent, A. I., \& Beckwith, S. V. W. 1993, Icarus, 106, 2

Malbet, F., Lachaume, R., \& Monin, J.-L. 2001, A\&A, 379, 515
Mannings, V., Koerner, D. W., \& Sargent, A. I. 1997, Nature, 388, 555 Monnier, J. D., \& Millan-Gabet, R. 2002, ApJ, 579, 694

Pavlyuchenkov, Y., Guilloteau, S., Henning, T., et al. 2007, in prep.

Pety, J. 2005, in SF2A-2005: Semaine de 1'Astrophysique Francaise, ed. F. Casoli, T. Contini, J. M. Hameury, \& L. Pagani, 721

Pety, J., Gueth, F., Guilloteau, S., \& Dutrey, A. 2006, A\&A, 458, 841

Piétu, V., Dutrey, A., \& Kahane, C. 2003, A\&A, 398, 565

Piétu, V., Dutrey, A., Guilloteau, S., Chapillon, E., \& Pety, J. 2006, A\&A, 460, L43

Piétu, V., Guilloteau, S., \& Dutrey, A. 2005, A\&A, 443, 945

Qi, C., Kessler, J. E., Koerner, D. W., Sargent, A. I., \& Blake, G. A. 2003, ApJ, 597, 986

Semenov, D., Pavlyuchenkov, Y., Schreyer, K., et al. 2005, ApJ, 621, 853

Semenov, D., Wiebe, D., \& Henning, T. 2006, ApJ, 647, L57

Siess, L., Dufour, E., \& Forestini, M. 2000, A\&A, 358, 593

Simon, M., Dutrey, A., \& Guilloteau, S. 2000, ApJ, 545, 1034

van den Ancker, M. E., de Winter, D., \& Tjin A Djie, H. R. E. 1998, A\&A, 330, 145

van Zadelhoff, G.-J., Aikawa, Y., Hogerheijde, M. R., \& van Dishoeck, E. F. 2003, A\&A, 397, 789

Willacy, K., Langer, W., Allen, M., \& Bryden, G. 2006, ApJ, 644, 1202 